\title{
Exponential Stabilization for Timoshenko Beam with Distributed Delay in the Boundary Control
}

\author{
Xiu Fang Liu and Gen Qi Xu \\ Department of Mathematics, Tianjin University, Tianjin 300072, China \\ Correspondence should be addressed to Xiu Fang Liu; liuxiu31@126.com
}

Received 23 October 2013; Accepted 18 November 2013

Academic Editor: Shen Yin

Copyright ( 2013 X. F. Liu and G. Q. Xu. This is an open access article distributed under the Creative Commons Attribution License, which permits unrestricted use, distribution, and reproduction in any medium, provided the original work is properly cited.

We consider the exponential stabilization for Timoshenko beam with distributed delay in the boundary control. Suppose that the controller outputs are of the form $\alpha_{1} u_{1}(t)+\beta_{1} u_{1}(t-\tau)+\int_{-\tau}^{0} g_{1}(\eta) u_{1}(t+\eta) d \eta$ and $\alpha_{2} u_{2}(t)+\beta_{2} u_{2}(t-\tau)+\int_{-\tau}^{0} g_{2}(\eta) u_{2}(t+\eta) d \eta$; where $u_{1}(t)$ and $u_{2}(t)$ are the inputs of boundary controllers. In the past, most stabilization results for wave equations and Euler-Bernoulli beam with delay are required $\alpha_{i}>\beta_{i}>0, i=1,2$. In the present paper, we will give the exponential stabilization about Timoshenko beam with distributed delay and demand to satisfy the lesser conditions for $\alpha_{i}, \beta_{i}, i=1,2$.

\section{Introduction}

Since the extensive applications of Timoshenko beam in high-Tech, the stabilization problem has been a hot topic in the mathematical control theory and engineering; for instance, see [1-5] and the references therein. In many literature, the control delay problem has been neglected. Due to extensive applications of the system with delay, more and more scholars devoted to study the stabilization of the system with controller delay. It is well known that time delay caused by controller memory usually takes the form $\int_{-\infty}^{0} d \alpha(s) u(t+$ $s)$, where $\alpha(s)$ is a bounded variation function (or matrixvalued function) and $u(t)$ is the control input. If the control is in the space $L_{\text {loc }}^{2}(\mathbb{R})$, then the memory controller will take the form

$$
\begin{aligned}
\int_{-\tau}^{0} d \alpha(s) u(t+s)= & \alpha u(t)+\beta u(t-\tau) \\
& +\int_{-\tau}^{0} g(s) u(t+s) d s .
\end{aligned}
$$

Based on this reason, Xu et al. (see [6]) studied firstly stabilization of the 1-d wave systems with delay of the form $\alpha u(t)+\beta u(t-\tau)$. They proved that the system with control delay is exponential stable if $\alpha>\beta>0$ and unstable if $\beta>\alpha$. Nicaise and Pignotti in [7] studied the stability and instability of the wave equation with delay in boundary and internal distributed delay. Nicaise and Valein in [8] extend the 1-d wave equation to the networks of 1-d wave equations. Shang et al. in [9] studied Euler-Bernoulli beam and showed that $\beta>0$ is not necessary, but the condition $\alpha>|\beta|$ is necessary. For the case of distributed delay, that is, $\beta>$ 0 and $\int_{-\tau}^{0}|g(\eta)| d \eta \neq 0$, Nicaise and Pignotti in [10] discussed a high dimensional wave equation. Under the condition that $\alpha>\int_{-\tau}^{0}|g(\eta)| d \eta \neq 0$, they proved the velocity feedback control law also stabilizes exponentially the system.

From above we see that $\alpha, \beta$, and $g(\eta)$ are determined by the controller. We cannot determine whether or not $\alpha>$ $\beta>0$ including $\alpha>|\beta|$ in practice. Under the assumption of state being measurable, Shang and $\mathrm{Xu}$ in [11] designed a dynamic feedback controller for cantilever EulerBernoulli beam that stabilizes exponentially the system for any real $|\alpha| \neq|\beta|$. Recently Han and Xu in [12] extended this result to the case of output being measurable; they showed that a state observer can realize the state reconstruction from the output of the system. Xu and Wang in [13] discussed the Timoshenko beam with boundary control delay, and they also stabilized the system by a dynamic feedback controller. 
Note that the difference between $[11,13]$, one is a system of single input and single output, the other is a system of 2 inputs and 2 outputs. Such discussion will lead us to extend the method to a general system of multiinput and multioutput. So far, however, there is no result for any $\alpha, \beta$, and $g(\eta)$ about Timoshenko beams. In this paper, we still consider Timoshenko beam with boundary control distributed delay. We will seek for a dynamic feedback control law that exponentially stabilizes the Timoshenko beam with distributed delay under certain conditions.

The rest is organized as follows. In Section 2, we will describe the design process of controllers, including predict system and generation of signal, and then state the main results of this paper. In Section 3, we will give the representation of the transform system. In Section 4, we will prove our first result on the stabilization of the original system. In Section 5, we will prove the second result on the exponential stabilization of the induced system. In Section 6, we conclude the paper.

\section{Design of Controllers and Main Results}

Let $w(x, t)$ be the displacement and $\varphi(x, t)$ the rotation angle of the beam. The motion of a cantilever beam is governed by the following partial differential equations:

$$
\begin{aligned}
& \rho w_{t t}(x, t)-K\left(w_{x x}-\varphi_{x}\right)(x, t)=0, \\
& x \in(0,1), t>0 \text {, } \\
& I_{\rho} \varphi_{t t}(x, t)-E I \varphi_{x x}(x, t)-K\left(w_{x}-\varphi\right)(x, t)=0, \\
& x \in(0,1), t>0 \text {, } \\
& w(0, t)=\varphi(0, t)=0, \quad t>0, \\
& K\left(w_{x}-\varphi\right)(1, t)=v_{1}(t), \\
& E I \varphi_{x}(1, t)=v_{2}(t), \\
& w(x, 0)=w_{0}(x), \quad w_{t}(x, 0)=w_{1}(x), \\
& \varphi(x, 0)=\varphi_{0}(x), \quad \varphi_{t}(x, 0)=\varphi_{1}(x),
\end{aligned}
$$

where $v_{1}(t)$ and $v_{2}(t)$ are the control force and torque from the controllers, respectively. If the controllers have no memory, namely, $u_{j}(t)=v_{j}(t), j=1,2$, where $u_{j}(t)$ are controller inputs, this model had been studied in [14]. If the controllers have memory, then the Timoshenko beam became

$$
\begin{gathered}
\rho w_{t t}(x, t)-K\left(w_{x x}-\varphi_{x}\right)(x, t)=0, \\
x \in(0,1), t>0, \\
I_{\rho} \varphi_{t t}(x, t)-E I \varphi_{x x}(x, t)-K\left(w_{x}-\varphi\right)(x, t)=0, \\
x \in(0,1), t>0, \\
w(0, t)=\varphi(0, t)=0, \quad t>0,
\end{gathered}
$$

$$
\begin{aligned}
& K\left(w_{x}-\varphi\right)(1, t)=\alpha_{1} u_{1}(t)+\beta_{1} u_{1}(t-\tau) \\
& +\int_{-\tau}^{0} g_{1}(\eta) u_{1}(t+\eta) d \eta, \\
& E I \varphi_{x}(1, t)=\alpha_{2} u_{2}(t)+\beta_{2} u_{2}(t-\tau) \\
& +\int_{-\tau}^{0} g_{2}(\eta) u_{2}(t+\eta) d \eta \\
& w(x, 0)=w_{0}(x), \quad w_{t}(x, 0)=w_{1}(x), \\
& \varphi(x, 0)=\varphi_{0}(x), \quad \varphi_{t}(x, 0)=\varphi_{1}(x), \\
& u_{1}(\theta)=f_{1}(\theta), \quad u_{2}(\theta)=f_{2}(\theta), \quad \theta \in(-\tau, 0) \text {, }
\end{aligned}
$$

where $\tau$ is the delay time, $\alpha_{i}, \beta_{i} \in \mathbb{R}(i=1,2)$ are the controller parameters, and $g_{i}(\eta) \in L^{2}[-\tau, 0], j=1,2$, and $f_{i}(\theta), \theta \in(-\tau, 0)(i=1,2)$ are bounded measurable functions that are memory values of controllers. When $g_{j} \equiv$ $0, j=1,2,(3)$ is just the model in [13].

We suppose that the state of (3) is measurable; that is, $\left(w(x, t), \varphi(x, t), w_{t}(x, t), \varphi_{t}(x, t)\right)$ is measurable. We introduce an auxiliary system as follows:

$$
\begin{aligned}
& \widehat{w}_{s}(x, s, t)=\widehat{z}(x, s, t), \quad x \in(0,1), s \in(0, \tau), \\
& \widehat{\varphi}_{s}(x, s, t)=\widehat{\psi}(x, s, t), \quad x \in(0,1), s \in(0, \tau), \\
& \widehat{z}_{s}(x, s, t)=\frac{K}{\rho}\left(\widehat{w}_{x x}(x, s, t)-\widehat{\varphi}_{x}(x, s, t)\right), \\
& \widehat{\psi}_{s}(x, s, t)=\frac{E I}{I_{\rho}} \widehat{\varphi}_{x x}(x, s, t)+\frac{K}{I_{\rho}}\left(\widehat{w}_{x}(x, s, t)-\widehat{\varphi}(x, s, t)\right), \\
& \widehat{w}(0, s, t)=\widehat{\varphi}(0, s, t)=0, \quad s \in(0, \tau), \\
& K\left(\widehat{w}_{x}-\widehat{\varphi}\right)(1, s, t)=\beta_{1} u_{1}(t+s-\tau) \\
& +\int_{-\tau}^{-s} g_{1}(\eta) u_{1}(t+s+\eta) d \eta \\
& s \in(0, \tau), \\
& E I \widehat{\varphi}_{x}(1, s, t)=\beta_{2} u_{2}(t+s-\tau) \\
& +\int_{-\tau}^{-s} g_{2}(\eta) u_{2}(t+s+\eta) d \eta \\
& s \in(0, \tau), t>0, \\
& \widehat{w}(x, 0, t)=w(x, t), \quad \widehat{z}(x, 0, t)=w_{t}(x, t), \\
& x \in(0,1), \\
& \widehat{\varphi}(x, 0, t)=\varphi(x, t), \quad \widehat{\psi}_{t}(x, 0, t)=\varphi_{t}(x, t), \\
& x \in(0,1), t>0 \text {. }
\end{aligned}
$$

Equation (4) is a partial state predictor. 
Denote the state of (4) at the moment $s=\tau$ by

$$
\begin{aligned}
& \left(p_{1}(x, t), p_{2}(x, t), q_{1}(x, t), q_{2}(x, t)\right) \\
& \quad=(\widehat{w}(x, \tau, t), \widehat{\varphi}(x, \tau, t), \widehat{z}(x, \tau, t), \widehat{\psi}(x, \tau, t)) .
\end{aligned}
$$

Using (3) we can verify that the functions group $\left(p_{1}(x, t)\right.$, $\left.p_{2}(x, t), q_{1}(x, t), q_{2}(x, t)\right)$ satisfy the following partial differential equations:

$$
\begin{aligned}
& p_{1, t}(x, t)=q_{1}(x, t)+a_{1}(x) u_{1}(t)+a_{2}(x) u_{2}(t), \\
& x \in(0,1), t>0, \\
& p_{2, t}(x, t)=q_{2}(x, t)+a_{3}(x) u_{1}(t)+a_{4}(x) u_{2}(t), \\
& x \in(0,1), t>0 \text {, } \\
& q_{1, t}(x, t)=\frac{K}{\rho}\left(p_{1, x x}-p_{2, x}\right)(x, t)+b_{1}(x) u_{1}(t) \\
& +b_{2}(x) u_{2}(t) \\
& q_{2, t}(x, t)=\frac{E I}{I_{\rho}} p_{2, x x}(x, t)+\frac{K}{I_{\rho}}\left(p_{1, x}-p_{2}\right)(x, t) \\
& +b_{3}(x) u_{1}(t)+b_{4}(x) u_{2}(t), \\
& p_{1}(0, t)=p_{2}(0, t)=q_{1}(0, t)=q_{2}(0, t)=0, \\
& t>0, \\
& K\left(p_{1, x}-p_{2}\right)(1, t)=\beta_{1} u_{1}(t), \quad t>0, \\
& \operatorname{EIp}_{2, x}(1, t)=\beta_{2} u_{2}(t), \quad t>0, \\
& p_{1}(x, 0)=E_{1}\left(w_{0}, \varphi_{0}, w_{1}, \varphi_{1}\right)(x)-\int_{-\tau}^{0} a_{1}(x, s) f_{1}(s) d s \\
& -\int_{-\tau}^{0} a_{2}(x, s) f_{2}(s) d s \\
& p_{2}(x, 0)=E_{2}\left(w_{0}, \varphi_{0}, w_{1}, \varphi_{1}\right)(x)-\int_{-\tau}^{0} a_{3}(x, s) f_{1}(s) d s \\
& -\int_{-\tau}^{0} a_{4}(x, s) f_{2}(s) d s \\
& q_{1}(x, 0)=E_{3}\left(w_{0}, \varphi_{0}, w_{1}, \varphi_{1}\right)(x)+\int_{-\tau}^{0} b_{1}(x, s) f_{1}(s) d s \\
& +\int_{-\tau}^{0} b_{2}(x, s) f_{2}(s) d s \\
& q_{2}(x, 0)=E_{4}\left(w_{0}, \varphi_{0}, w_{1}, \varphi_{1}\right)(x)+\int_{-\tau}^{0} b_{3}(x, s) f_{1}(s) d s \\
& +\int_{-\tau}^{0} b_{4}(x, s) f_{2}(s) d s
\end{aligned}
$$

where $a_{i}(x, r), b_{i}(x, r), a_{i}(x), b_{i}(x)(i=1,2,3,4)$ are measurable function and $E_{i}(i=1,2,3,4)$ are bounded linear operators on $\left[H^{1}[0,1] \times L^{2}[0,1]\right]^{2}$; they are determined later.
Equation (6) is a system without delay, but the controls appear in the system interior and boundary. First we consider the stabilization problem of (6). Let us consider the energy functional of (6)

$$
\begin{aligned}
E(t)= & \frac{1}{2}\left\|\left(p_{1}, p_{2}\right)\right\|_{V_{K}^{1}(0,1) \times V_{E I}^{1}(0,1)}^{2} \\
& +\frac{1}{2}\left\|\left(q_{1}, q_{2}\right)\right\|_{L_{\rho}^{2}(0,1) \times L_{I_{\rho}}^{2}(0,1)}^{2} \\
= & \frac{1}{2} \int_{0}^{1} K\left|p_{1, x}(x, t)-p_{2}(x, t)\right|^{2} d x \\
& +\frac{1}{2} \int_{0}^{1} E I\left|p_{2, x}(x, t)\right|^{2} d x \\
& +\frac{1}{2} \int_{0}^{1} \rho\left|q_{1}(x, t)\right|^{2} d x \\
& +\frac{1}{2} \int_{0}^{1} I_{\rho}\left|q_{2}(x, t)\right|^{2} d x .
\end{aligned}
$$

A direct calculation gives

$$
\begin{aligned}
& \frac{d E(t)}{d t} \\
& =u_{1}(t)\left[\beta_{1} q_{1}(1, t)\right. \\
& +\int_{0}^{1} K\left(p_{1, x}-p_{2}\right)\left[a_{1}^{\prime}(x)-a_{3}(x)\right] d x \\
& +\int_{0}^{1} \operatorname{EIp}_{2, x}(x, t) a_{3}^{\prime}(x) d x \\
& +\int_{0}^{1} \rho q_{1}(x, t) b_{1}(x) d x \\
& \left.+\int_{0}^{1} I_{\rho} q_{2}(x, t) b_{3}(x) d x\right] \\
& +u_{2}(t)\left[\beta_{2} q_{2}(1, t)+\int_{0}^{1} K\left(p_{1, x}-p_{2}\right)\right. \\
& \times\left[a_{2}^{\prime}(x)-a_{4}(x)\right] d x \\
& +\int_{0}^{1} \operatorname{EIp}_{2, x}(x, t) a_{4}^{\prime}(x) d x \\
& +\int_{0}^{1} \rho q_{1}(x, t) b_{2}(x) d x \\
& \left.+\int_{0}^{1} I_{\rho} q_{2}(x, t) b_{4}(x) d x\right] \text {. }
\end{aligned}
$$

Set

$$
\begin{aligned}
U_{1} & \left(p_{1}, p_{2}, q_{1}, q_{2}\right) \\
= & \beta_{1} q_{1}(1, t) \\
& \quad+\int_{0}^{1} K\left(p_{1, x}(x, t)-p_{2}(x, t)\right)\left(a_{1}^{\prime}(x)-a_{3}(x)\right) d x
\end{aligned}
$$




$$
\begin{aligned}
&+\int_{0}^{1} \rho q_{1}(x, t) b_{1}(x) d x+\int_{0}^{1} \operatorname{EI}_{2, x}(x, t) a_{3}^{\prime}(x) d x \\
&+\int_{0}^{1} I_{\rho} q_{2}(x, t) b_{3}(x) d x \\
& U_{2}\left(p_{1}, p_{2}, q_{1}, q_{2}\right) \\
&=\beta_{2} q_{2}(1, t) \\
& \quad+\int_{0}^{1} K\left(p_{1, x}(x, t)-p_{2}(x, t)\right)\left(a_{2}^{\prime}(x)-a_{4}(x)\right) d x \\
&+\int_{0}^{1} \rho q_{1}(x, t) b_{2}(x) d x+\int_{0}^{1} \operatorname{EI}_{2, x}(x, t) a_{4}^{\prime}(x) d x \\
&+\int_{0}^{1} I_{\rho} q_{2}(x, t) b_{4}(x) d x .
\end{aligned}
$$

We take the feedback control law as

$$
\begin{aligned}
& u_{1}(t)=-U_{1}\left(p_{1}, p_{2}, q_{1}, q_{2}\right), \\
& u_{2}(t)=-U_{2}\left(p_{1}, p_{2}, q_{1}, q_{2}\right) .
\end{aligned}
$$

Then, the closed loop system associated with (6) is

$$
\begin{aligned}
& p_{1, t}(x, t)=q_{1}(x, t)-a_{1}(x) U_{1}\left(p_{1}, p_{2}, q_{1}, q_{2}\right) \\
& -a_{2}(x) U_{2}\left(p_{1}, p_{2}, q_{1}, q_{2}\right) \text {, } \\
& x \in(0,1), t>0, \\
& p_{2, t}(x, t)=q_{2}(x, t)-a_{3}(x) U_{1}\left(p_{1}, p_{2}, q_{1}, q_{2}\right) \\
& -a_{4}(x) U_{2}\left(p_{1}, p_{2}, q_{1}, q_{2}\right) \text {, } \\
& x \in(0,1), t>0, \\
& q_{1, t}(x, t)=\frac{K}{\rho}\left(p_{1, x x}-p_{2, x}\right)(x, t)-b_{1}(x) U_{1} \\
& \times\left(p_{1}, p_{2}, q_{1}, q_{2}\right)-b_{2}(x) U_{2}\left(p_{1}, p_{2}, q_{1}, q_{2}\right), \\
& q_{2, t}(x, t)=\frac{E I}{I_{\rho}} p_{2, x x}(x, t)+\frac{K}{I_{\rho}}\left(p_{1, x}-p_{2}\right)(x, t) \\
& -b_{3}(x) U_{1}\left(p_{1}, p_{2}, q_{1}, q_{2}\right) \\
& -b_{4}(x) U_{2}\left(p_{1}, p_{2}, q_{1}, q_{2}\right) \text {, } \\
& p_{1}(0, t)=p_{2}(0, t)=q_{1}(0, t)=q_{2}(0, t)=0, \quad t>0, \\
& K\left(p_{1, x}-p_{2}\right)(1, t)=-\beta_{1} U_{1}\left(p_{1}, p_{2}, q_{1}, q_{2}\right), \quad t>0, \\
& E I p_{2, x x}(1, t)=-\beta_{2} U_{2}\left(p_{1}, p_{2}, q_{1}, q_{2}\right), \quad t>0, \\
& p_{1}(x, 0)=E_{1}\left(w_{0}, \varphi_{0}, w_{1}, \varphi_{1}\right)(x)-\int_{-\tau}^{0} a_{1}(x, s) f_{1}(s) d s \\
& -\int_{-\tau}^{0} a_{2}(x, s) f_{1}(s) d s
\end{aligned}
$$

$$
\begin{aligned}
p_{2}(x, 0)= & E_{2}\left(w_{0}, \varphi_{0}, w_{1}, \varphi_{1}\right)(x)-\int_{-\tau}^{0} a_{3}(x, s) f_{1}(s) d s \\
& -\int_{-\tau}^{0} a_{4}(x, s) f_{1}(s) d s \\
q_{1}(x, 0)= & E_{3}\left(w_{0}, \varphi_{0}, w_{1}, \varphi_{1}\right)(x)+\int_{-\tau}^{0} b_{1}(x, s) f_{1}(s) d s \\
& +\int_{-\tau}^{0} b_{2}(x, s) f_{1}(s) d s, \\
q_{2}(x, 0)= & E_{4}\left(w_{0}, \varphi_{0}, w_{1}, \varphi_{1}\right)(x)+\int_{-\tau}^{0} b_{3}(x, s) f_{1}(s) d s \\
& +\int_{-\tau}^{0} b_{4}(x, s) f_{1}(s) d s .
\end{aligned}
$$

We estimate the error of the system (3) with control (10) and the system (11).

Let $\left(w(x, t), \varphi(x, t), w_{t}(x, t), \varphi_{t}(x, t)\right)$ be the solution to (3) with control signals (10) and let function group $\left(p_{1}(x, t)\right.$, $\left.p_{2}(x, t), q_{1}(x, t), q_{2}(x, t)\right)$ be the solution to (11). Set $W(x, t)$ $=(w(x, t), \varphi(x, t))$ and $W_{t}(x, t)=\left(w_{t}(x, t), \varphi_{t}(x, t)\right)$, and set $P(x, t)=\left(p_{1}(x, t), p_{2}(x, t)\right)$ and $Q(x, t)=\left(q_{1}(x, t)\right.$, $\left.q_{2}(x, t)\right)$.

To discuss the stability $\left(W(x, t), W_{t}(x, t)\right)$, we consider the error both solutions in the energy space

$$
\begin{aligned}
& \|P(\cdot, t)-W(\cdot, t+\tau)\|_{V_{K}^{1}(0,1) \times V_{E I}^{1}(0,1)}^{2} \\
& \quad+\left\|Q(\cdot, t)-W_{t}(\cdot, t+\tau)\right\|_{L_{\rho}^{2}(0,1) \times L_{I_{\rho}}^{2}(0,1)}^{2} .
\end{aligned}
$$

In this paper, we will prove the following results.

Theorem 1. Let $\left(W(x, t), W_{t}(x, t)\right)$ be the solution to (3) with controls (10) and let $(P(x, t), Q(x, t))$ be the solution to the closed-loop system (11). If the system (11) is asymptotically (exponentially) stable, then the system (3) also is asymptotically (exponentially) stable.

Theorem 2. Suppose that $K / \rho \neq E I / I_{\rho}$. Let $\mu_{n}, n \in \mathbb{N}$ be the eigenvalues of the free system (the system (2) without controls). Set

$$
\begin{aligned}
& \xi_{n}^{(1)}=\int_{-\tau}^{0} g_{1}(\eta) e^{-i \sqrt{\mu_{n}}(\tau+\eta)} d \eta, \\
& \xi_{n}^{(2)}=\int_{-\tau}^{0} g_{2}(\eta) e^{-i \sqrt{\mu_{n}}(\tau+\eta)} d \eta .
\end{aligned}
$$

Then the following assertions are true:

(1) when

$$
\begin{aligned}
& \inf _{n}\left|\frac{\beta_{1}}{\rho}+\alpha_{1} e^{-i \tau \sqrt{\mu_{n}}}+\xi_{n}^{(1)}\right|>0, \\
& \inf _{n}\left|\frac{\beta_{2}}{I_{\rho}}+\alpha_{2} e^{-i \tau \sqrt{\mu_{n}}}+\xi_{n}^{(2)}\right|>0,
\end{aligned}
$$

the system (11) is exponentially stable; 
(2) if for all $n \in \mathbb{N}$,

$$
\begin{aligned}
& \left|\frac{\beta_{1}}{\rho}+\alpha_{1} e^{-i \tau \sqrt{\mu_{n}}}+\xi_{n}^{(1)}\right|>0, \\
& \left|\frac{\beta_{2}}{I_{\rho}}+\alpha_{2} e^{-i \tau \sqrt{\mu_{n}}}+\xi_{n}^{(2)}\right|>0
\end{aligned}
$$

but

$$
\begin{aligned}
& \inf _{n}\left|\frac{\beta_{1}}{\rho}+\alpha_{1} e^{-i \tau \sqrt{\mu_{n}}}+\xi_{n}^{(1)}\right|=0, \\
& \inf _{n}\left|\frac{\beta_{2}}{I_{\rho}}+\alpha_{2} e^{-i \tau \sqrt{\mu_{n}}}+\xi_{n}^{(2)}\right|=0,
\end{aligned}
$$

then the system (11) is asymptotically stable.

In the following sections, we will prove our results. In Section 3, we will determine functions $a_{i}(x), b_{i}(x)$, $a_{i}(x, s), b_{i}(x, s)(i=1,2,3,4)$. In Section 4 , we will prove Theorem 1. In Section 5, we pay our attention to the proof of Theorem 2.

\section{Representation of the System (6)}

In this section, we will obtain the expressions for the functions $a_{i}(x), b_{i}(x), a_{i}(x, s), b_{i}(x, s)(i=1,2,3,4)$ appearing in system (6) using (3) and (4).

We begin with introducing two useful lemmas.

Lemma 3 (see [13]). Define the differential operator in $L_{\rho}^{2}(0,1) \times L_{I_{\rho}}^{2}(0,1)$ as follows:

$$
\begin{gathered}
\mathscr{L}(w, \varphi)=\left(-\frac{K}{\rho}\left(w^{\prime \prime}(x)-\varphi^{\prime}(x)\right),-\frac{E I}{I_{\rho}} \varphi^{\prime \prime}(x)\right. \\
\left.-\frac{K}{I_{\rho}}\left(w^{\prime}(x)-\varphi(x)\right)\right)^{T},
\end{gathered}
$$

with domain

$$
\begin{aligned}
& \mathscr{D}(\mathscr{L}) \\
& =\left\{\begin{array}{cc}
(w(x), \varphi(x)) \in H^{2}(0,1) & w(0)=\varphi(0)=0, \\
\times H^{2}(0,1) & K\left(w^{\prime}(1)-\varphi(1)\right)=0, \\
E I \varphi^{\prime}(1)=0
\end{array}\right\} .
\end{aligned}
$$

Then $\mathscr{L}$ is a positive define operator with compact resolvent in $L_{\rho}^{2}(0,1) \times L_{I_{\rho}}^{2}(0,1)$; its eigenvalues are

$$
0<\mu_{1}<\mu_{2}<\cdots<\mu_{n}<\cdots
$$

and the eigenfunctions $\Phi_{n}(x)=\left(w_{n}(x), \varphi_{n}(x)\right)^{T}$ corresponding to $\mu_{n}$ are real functions and form a normalized orthogonal basis for $L_{\rho}^{2}(0,1) \times L_{I_{\rho}}^{2}(0,1)$.
Lemma 4 (see [13]). Let $\Phi_{n}(x)=\left(w_{n}(x), \varphi_{n}(x)\right)$ be the normalized eigenfunction corresponding to the eigenvalue $\mu_{n}$ of $\mathscr{L}$. Then it holds that

$$
\begin{aligned}
\int_{0}^{1} K\left|\omega_{n}^{\prime}(x)-\varphi_{n}(x)\right|^{2} d x+\int_{0}^{1} E I\left|\varphi_{n}^{\prime}(x)\right|^{2} d x=\mu_{n} \\
0<\inf _{n}\left\{\rho\left|w_{n}(1)\right|^{2}+\left|I_{\rho} \varphi_{n}(1)\right|^{2}\right\} \\
\leq \sup _{n}\left\{\rho\left|w_{n}(1)\right|^{2}+\left|I_{\rho} \varphi_{n}(1)\right|^{2}\right\}<\infty .
\end{aligned}
$$

Now let us return to (3). We write the equation in (3) into the vector form

$$
\left(\begin{array}{l}
w_{t t}(x, t) \\
\varphi_{t t}(x, t)
\end{array}\right)-\left(\begin{array}{cc}
\frac{K}{\rho} \partial_{x x} & -\frac{K}{\rho} \partial_{x} \\
\frac{K}{I_{\rho}} \partial_{x} & \frac{E I}{I_{\rho}} \partial_{x x}-\frac{K}{I_{\rho}}
\end{array}\right)\left(\begin{array}{l}
w(x, t) \\
\varphi(x, t)
\end{array}\right)=0
$$

and the boundary conditions are $\left(\begin{array}{c}w(0, t) \\ \varphi(0, t)\end{array}\right)=0$, and

$$
\begin{aligned}
\left(\begin{array}{cc}
K \partial_{x} & -K \\
0 & E I \partial_{x}
\end{array}\right)\left(\begin{array}{l}
w(x, t) \\
\varphi(x, t)
\end{array}\right)_{x=1} \\
\quad=\left(\begin{array}{cc}
\alpha_{1} & 0 \\
0 & \alpha_{2}
\end{array}\right)\left(\begin{array}{l}
u_{1}(t) \\
u_{2}(t)
\end{array}\right)+\left(\begin{array}{cc}
\beta_{1} & 0 \\
0 & \beta_{2}
\end{array}\right)\left(\begin{array}{l}
u_{1}(t-\tau) \\
u_{2}(t-\tau)
\end{array}\right) \\
\quad+\int_{-\tau}^{0}\left(\begin{array}{cc}
g_{1}(\eta) & 0 \\
0 & g_{2}(\eta)
\end{array}\right)\left(\begin{array}{l}
u_{1}(t+\eta) \\
u_{2}(t+\eta)
\end{array}\right) d \eta .
\end{aligned}
$$

The initial datum are

$$
\left(\begin{array}{l}
w(x, 0) \\
\varphi(x, 0)
\end{array}\right)=\left(\begin{array}{l}
w_{0}(x) \\
\varphi_{0}(x)
\end{array}\right), \quad\left(\begin{array}{l}
w_{t}(x, 0) \\
\varphi_{t}(x, 0)
\end{array}\right)=\left(\begin{array}{l}
w_{1}(x) \\
\varphi_{1}(x)
\end{array}\right) .
$$

Set $W(x, t)=(w(x, t), \varphi(x, t))^{T}$ and $U(t)=\left(u_{1}(t), u_{2}(t)\right)^{T}$. Define $2 \times 2$ matrices

$$
\begin{gathered}
\Lambda_{1}=\left(\begin{array}{cc}
\alpha_{1} & 0 \\
0 & \alpha_{2}
\end{array}\right), \quad \Lambda_{2}=\left(\begin{array}{cc}
\beta_{1} & 0 \\
0 & \beta_{2}
\end{array}\right), \\
\Lambda_{3}(\eta)=\left(\begin{array}{cc}
g_{1}(\eta) & 0 \\
0 & g_{2}(\eta)
\end{array}\right)
\end{gathered}
$$

and define an operator $B$ from $\mathbb{R}^{2}$ to $H^{-1}(0,1) \times H^{-1}(0,1)$, where $H^{-1}(0,1)=\left(V_{\omega}^{1}(0,1)\right)^{*}$ is dual space,

$$
B=\left(\begin{array}{cc}
\delta(x-1) & 0 \\
0 & \delta(x-1)
\end{array}\right),
$$

and define an operator $\Gamma_{N}$ from $H^{2}(0,1) \times H^{2}(0,1)$ to $\mathbb{R}^{2}$ by

$$
\Gamma_{N} W=\left(\begin{array}{c}
K\left(w^{\prime}(1)-\varphi(1)\right) \\
E I \varphi^{\prime}(1)
\end{array}\right),
$$

where $W(x)=(w(x), \varphi(x))^{T}$. 
With help of these notations, we can rewrite (3) into

$$
\begin{aligned}
& W_{t t}(x, t)+\mathscr{L} W(x, t) \\
& =B\left(\Lambda_{1} U(t)+\Lambda_{2} U(t-\tau)+\int_{-\tau}^{0} \Lambda_{3}(\eta) U(t+\eta) d \eta\right) \\
& t>0,
\end{aligned}
$$

$W(0, t)=0, \quad \Gamma_{N} W(1, t)=0$,

$$
\begin{aligned}
& W(x, 0)=W_{0}(x)=\left(w_{0}(x), \varphi_{0}(x)\right)^{T}, \\
& W_{t}(x, 0)=W_{1}(x)=\left(w_{1}(x), \varphi_{1}(x)\right)^{T},
\end{aligned}
$$

and (4) into

$$
\begin{gathered}
\widehat{W}_{s}(x, s, t)=\widehat{V}(x, s, t), \\
\widehat{V}_{s}(x, s, t)+\mathscr{L} \widehat{W}(x, s, t)=B \Lambda_{2} U(t+s-\tau) \\
+B \int_{-\tau}^{-s} \Lambda_{3}(\eta) U(t+s+\eta) d \eta, \\
\widehat{W}(0, s, t)=0, \quad \Gamma_{N} \widehat{W}(1, s, t)=0, \\
\widehat{W}(x, 0, t)=W(x, t), \\
\widehat{V}(x, 0, t)=W_{t}(x, t),
\end{gathered}
$$

where $\widehat{W}(x, s, t)=(\widehat{w}(x, s, t), \widehat{\varphi}(x, s, t))^{T}, \widehat{V}(x, s, t)=$ $(\widehat{z}(x, s, t), \widehat{\psi}(x, s, t))^{T}$.

We define two families of the bounded linear operators on $L_{\rho}^{2}(0,1) \times L_{I_{\rho}}^{2}(0,1)$ by

$$
\begin{aligned}
& \operatorname{Cos}(t \mathscr{L}) F=\sum_{n=1}^{\infty} \cos \sqrt{\mu_{n}} t\left(F, \Phi_{n}\right)_{L_{\rho}^{2} \times L_{I_{\rho}}^{2}} \Phi_{n}, \\
& \operatorname{Sin}(t \mathscr{L}) F=\sum_{n=1}^{\infty} \frac{\sin \sqrt{\mu_{n}} t}{\sqrt{\mu_{n}}}\left(F, \Phi_{n}\right)_{L_{\rho}^{2} \times L_{I_{\rho}}^{2}} \Phi_{n} .
\end{aligned}
$$

Clearly, the following equalities hold, for any $t \in \mathbb{R}$,

$$
\begin{gathered}
\operatorname{Sin}(t \mathscr{L})=\int_{0}^{t} \operatorname{Cos}(t \mathscr{L}) d t, \\
\frac{d}{d t}(\operatorname{Cos}(t \mathscr{L}))=-\mathscr{L} \operatorname{Sin}(t \mathscr{L}) .
\end{gathered}
$$

It is easy to know that the vector-valued function

$$
\begin{aligned}
W(x, t)= & \operatorname{Cos}(t \mathscr{L}) W_{0}+\operatorname{Sin}(t \mathscr{L}) W_{1} \\
& +\int_{0}^{t} \operatorname{Sin}((t-s) \mathscr{L}) B\left[\Lambda_{1} U(s)+\Lambda_{2} U(s-\tau)\right] d s \\
& +\int_{0}^{t} \operatorname{Sin}((t-s) \mathscr{L}) B \int_{-\tau}^{0} \Lambda_{3}(\eta) U(s+\eta) d \eta d s
\end{aligned}
$$

is differentiable with respect to $t$ and

$$
\begin{aligned}
W_{t}(x, t)= & -\mathscr{L} \operatorname{Sin}(t \mathscr{L}) W_{0}+\operatorname{Cos}(t \mathscr{L}) W_{1} \\
& +\int_{0}^{t} \operatorname{Cos}((t-s) \mathscr{L}) B\left[\Lambda_{1} U(s)+\Lambda_{2} U(s-\tau)\right] d s \\
& +\int_{0}^{t} \operatorname{Cos}((t-s) \mathscr{L}) B \int_{-\tau}^{0} \Lambda_{3}(\eta) U(s+\eta) d \eta d s .
\end{aligned}
$$

Further, $W(x, t)$ satisfies (27).

Similarly, we know the vector-valued function

$$
\begin{aligned}
\widehat{W}(x, s, t) \\
=\operatorname{Cos}(s \mathscr{L}) W(\cdot, t)+\operatorname{Sin}(s \mathscr{L}) W_{t}(\cdot, t) \\
\quad+\int_{0}^{s} \operatorname{Sin}((s-r) \mathscr{L}) B \Lambda_{2} U(t+r-\tau) d r \\
\quad+\int_{0}^{s} \operatorname{Sin}((s-r) \mathscr{L}) B \int_{-\tau}^{-r} \Lambda_{3}(\eta) U(t+r+\eta) d \eta d r,
\end{aligned}
$$

$\widehat{V}(x, s, t)$

$$
\begin{aligned}
= & -\mathscr{L} \operatorname{Sin}(s \mathscr{L}) W(\cdot, t)+\operatorname{Cos}(s \mathscr{L}) W_{t}(\cdot, t) \\
& +\int_{0}^{s} \operatorname{Cos}((s-r) \mathscr{L}) B \Lambda_{2} U(t+r-\tau) d r \\
& +\int_{0}^{s} \operatorname{Cos}((s-r) \mathscr{L}) B \int_{-\tau}^{-r} \Lambda_{3}(\eta) U(t+r+\eta) d \eta d r .
\end{aligned}
$$

satisfy (28).

Set

$$
P(x, t)=\widehat{W}(x, \tau, t), \quad Q(x, t)=\widehat{V}(x, \tau, t) .
$$

Then we have

$$
\begin{aligned}
\left(\begin{array}{c}
P(x, t) \\
Q(x, t)
\end{array}\right)= & \left(\begin{array}{cc}
\operatorname{Cos}(\tau \mathscr{L}) & \operatorname{Sin}(\tau \mathscr{L}) \\
-\mathscr{L} \operatorname{Sin}(\tau \mathscr{L}) & \operatorname{Cos}(\tau \mathscr{L})
\end{array}\right)\left(\begin{array}{c}
W(x, t) \\
W_{t}(x, t)
\end{array}\right) \\
& +\int_{0}^{\tau}\left(\begin{array}{c}
\operatorname{Sin}((\tau-r) \mathscr{L}) \\
\operatorname{Cos}((\tau-r) \mathscr{L})
\end{array}\right) B \Lambda_{2} U(t+r-\tau) d r \\
& +\int_{0}^{\tau}\left(\begin{array}{c}
\operatorname{Sin}((\tau-r) \mathscr{L}) \\
\operatorname{Cos}((\tau-r) \mathscr{L})
\end{array}\right) \\
& \times B \int_{-\tau}^{-r} \Lambda_{3}(\eta) U(t+r+\eta) d \eta d r .
\end{aligned}
$$

Thus,

$$
\begin{aligned}
&\left(\begin{array}{c}
P_{t}(x, t) \\
Q_{t}(x, t)
\end{array}\right)=\left(\begin{array}{cc}
\operatorname{Cos}(\tau \mathscr{L}) & \operatorname{Sin}(\tau \mathscr{L}) \\
-\mathscr{L} \operatorname{Sin}(\tau \mathscr{L}) & \operatorname{Cos}(\tau \mathscr{L})
\end{array}\right)\left(\begin{array}{c}
W_{t}(x, t) \\
W_{t t}(x, t)
\end{array}\right) \\
&+\int_{0}^{\tau}\left(\begin{array}{c}
\operatorname{Cos}((\tau-r) \mathscr{L}) \\
-\mathscr{L} \operatorname{Sin}((\tau-r) \mathscr{L})
\end{array}\right) \\
& \times B \Lambda_{2} U(t+r-\tau) d r
\end{aligned}
$$




$$
\begin{array}{ll}
+\left(\begin{array}{c}
0 \\
B \Lambda_{2} U(t)
\end{array}\right)-\left(\begin{array}{c}
\operatorname{Sin}(\tau \mathscr{L}) \\
\operatorname{Cos}(\tau \mathscr{L})
\end{array}\right) B \Lambda_{2} U(t-\tau) & \text { Note that } \\
-\left(\begin{array}{c}
\operatorname{Sin}(\tau \mathscr{L}) \\
\operatorname{Cos}(\tau \mathscr{L})
\end{array}\right) B \int_{-\tau}^{0} \Lambda_{3}(\eta) U(t+\eta) d \eta & \left(\begin{array}{c}
W_{t}(x, t) \\
W_{t t}(x, t)
\end{array}\right) \\
+\int_{0}^{\tau}\left(\begin{array}{c}
\operatorname{Cos}((\tau-r) \mathscr{L}) \\
-\mathscr{L} \operatorname{Sin}((\tau-r) \mathscr{L})
\end{array}\right) B & =\left(\begin{array}{cc}
0 & I \\
-\mathscr{L} & 0
\end{array}\right)\left(\begin{array}{c}
W(x, t) \\
W_{t}(x, t)
\end{array}\right) \\
& +\left(\begin{array}{c}
{ }^{-r} \Lambda_{3}(\eta) U(t+r+\eta) d \eta d r \\
\left.\left.\int_{-\tau} U(t)+\Lambda_{2} U(t-\tau)+\int_{-\tau}^{0} \Lambda_{3}(\eta) U(t+\eta) d \eta\right)\right)
\end{array}\right) \\
+\int_{-\tau}^{0}\left(\begin{array}{c}
\operatorname{Sin}((\tau+\eta) \mathscr{L}) \\
\operatorname{Cos}((\tau+\eta) \mathscr{L})
\end{array}\right) B \Lambda_{3}(\eta) U(t) d \eta . &
\end{array}
$$

(37) So it holds that

$$
\begin{aligned}
\left(\begin{array}{l}
P_{t}(x, t) \\
Q_{t}(x, t)
\end{array}\right)= & \left(\begin{array}{cc}
0 & I \\
-\mathscr{L} & 0
\end{array}\right)\left(\begin{array}{l}
P(x, t) \\
Q(x, t)
\end{array}\right) \\
& +\left(\begin{array}{c}
\operatorname{Sin}(\tau \mathscr{L}) B \Lambda_{1} U(t)+\int_{-\tau}^{0} \operatorname{Sin}((\tau+\eta) \mathscr{L}) B \Lambda_{3}(\eta) U(t) d \eta \\
\operatorname{Cos}(\tau \mathscr{L}) B \Lambda_{1} U(t)+\int_{-\tau}^{0} \operatorname{Cos}((\tau+\eta) \mathscr{L}) B \Lambda_{3}(\eta) U(t) d \eta+B \Lambda_{2} U(t)
\end{array}\right) .
\end{aligned}
$$

Therefore, we have equations

$$
\begin{aligned}
P_{t}(x, t)= & Q(x, t)+\operatorname{Sin}(\tau \mathscr{L}) B \Lambda_{1} U(t) \\
& +\int_{-\tau}^{0} \operatorname{Sin}((\tau+\eta) \mathscr{L}) B \Lambda_{3}(\eta) U(t) d \eta, \\
Q_{t}(x, t)= & -\mathscr{L} P(x, t)+\operatorname{Cos}(\tau \mathscr{L}) B \Lambda_{1} U(t) \\
& +\int_{-\tau}^{0} \operatorname{Cos}((\tau+\eta) \mathscr{L}) B \Lambda_{3}(\eta) U(t) d \eta \\
P(0, t)= & Q(0, t)=0, \quad \Gamma_{N} P(\cdot, t)=\Lambda_{2} U(t)
\end{aligned}
$$

and initial conditions

$$
\begin{aligned}
P(x, 0)= & \operatorname{Cos}(\tau \mathscr{L}) W_{0}+\operatorname{Sin}(\tau \mathscr{L}) W_{1} \\
& -\int_{-\tau}^{0} \operatorname{Sin}(s \mathscr{L}) B \Lambda_{2} f(s) d s \\
& +\int_{-\tau}^{0} \int_{-\tau}^{s} \operatorname{Sin}((\tau-s+\eta) \mathscr{L}) \\
& \times B \Lambda_{3}(\eta) f(s) d \eta d s,
\end{aligned}
$$$$
Q(x, 0)=-\mathscr{L} \operatorname{Sin}(\tau \mathscr{L}) W_{0}+\operatorname{Cos}(\tau \mathscr{L}) W_{1}
$$$$
+\int_{-\tau}^{0} \operatorname{Cos}(s \mathscr{L}) B \Lambda_{2} f(s) d s
$$$$
+\int_{-\tau}^{0} \int_{-\tau}^{s} \operatorname{Cos}((\tau-s+\eta) \mathscr{L}) B \Lambda_{3}(\eta) f(s) d \eta d s,
$$

where $f(s)=\left(f_{1}(s), f_{2}(s)\right)^{T}$.
Since all entries of $B$ are meaningful as linear functional on $H^{1}(0,1)$, so for any $Z=\left(z_{1}, z_{2}\right) \in \mathbb{R}^{2}$ and $\Phi_{n}(x) \in$ $H^{1}(0,1) \times H^{1}(0,1)$,

$$
\begin{aligned}
& \left(B Z, \Phi_{n}\right)_{L_{\rho}^{2}(0,1) \times L_{I_{\rho}}^{2}(0,1)} \\
& \quad=z_{1} \int_{0}^{1} \rho \delta(x-1) w_{n}(x) d x+z_{2} \int_{0}^{1} I_{\rho} \delta(x-1) \varphi_{n}(x) d x \\
& \quad=\rho z_{1} w_{n}(1)+I_{\rho} z_{2} \varphi_{n}(1)=\left[z_{1}, z_{2}\right]\left[\rho w_{n}(1), \varphi_{n}(1)\right]^{T}
\end{aligned}
$$

Therefore, we have the following results.

Theorem 5. Let $\left\{\mu_{n} ; n \in \mathbb{N}\right\}$ be the list of all eigenvalues of $\mathscr{L}$. Then the functions that appear in (6) are

$$
\begin{aligned}
& a_{1}(x)=\rho \sum_{n=1}^{\infty} w_{n}(1) w_{n}(x) \\
& \times\left(\alpha_{1} \frac{\sin \tau \sqrt{\mu_{n}}}{\sqrt{\mu_{n}}}\right. \\
& \left.+\int_{-\tau}^{0} g_{1}(\eta) \frac{\sin (\tau+\eta) \sqrt{\mu_{n}}}{\sqrt{\mu_{n}}} d \eta\right), \\
& a_{2}(x)=I_{\rho} \sum_{n=1}^{\infty} \varphi_{n}(1) w_{n}(x) \\
& \times\left(\alpha_{2} \frac{\sin \tau \sqrt{\mu_{n}}}{\sqrt{\mu_{n}}}\right. \\
& \left.+\int_{-\tau}^{0} g_{2}(\eta) \frac{\sin (\tau+\eta) \sqrt{\mu_{n}}}{\sqrt{\mu_{n}}} d \eta\right),
\end{aligned}
$$


8

Abstract and Applied Analysis

$$
\begin{aligned}
& a_{3}(x)=\rho \sum_{n=1}^{\infty} w_{n}(1) \varphi_{n}(x) \\
& a_{2}(x, s)=I_{\rho} \sum_{n=1}^{\infty} \varphi_{n}(1) w_{n}(x) \\
& \times\left(\alpha_{1} \frac{\sin \tau \sqrt{\mu_{n}}}{\sqrt{\mu_{n}}}\right. \\
& \times\left(\beta_{2} \frac{\sin s \sqrt{\mu_{n}}}{\sqrt{\mu_{n}}}\right. \\
& \left.+\int_{-\tau}^{0} g_{1}(\eta) \frac{\sin (\tau+\eta) \sqrt{\mu_{n}}}{\sqrt{\mu_{n}}} d \eta\right), \\
& \left.-\int_{-\tau}^{s} g_{2}(\eta) \frac{\sin (\tau-s+\eta) \sqrt{\mu_{n}}}{\sqrt{\mu_{n}}} d \eta\right), \\
& a_{3}(x, s)=\rho \sum_{n=1}^{\infty} w_{n}(1) \varphi_{n}(x) \\
& a_{4}(x)=I_{\rho} \sum_{n=1}^{\infty} \varphi_{n}(1) \varphi_{n}(x) \\
& \times\left(\beta_{1} \frac{\sin s \sqrt{\mu_{n}}}{\sqrt{\mu_{n}}}\right. \\
& \times\left(\alpha_{2} \frac{\sin \tau \sqrt{\mu_{n}}}{\sqrt{\mu_{n}}}\right. \\
& \left.-\int_{-\tau}^{s} g_{1}(\eta) \frac{\sin (\tau-s+\eta) \sqrt{\mu_{n}}}{\sqrt{\mu_{n}}} d \eta\right), \\
& \left.+\int_{-\tau}^{0} g_{2}(\eta) \frac{\sin (\tau+\eta) \sqrt{\mu_{n}}}{\sqrt{\mu_{n}}} d \eta\right) \text {, } \\
& b_{1}(x)=\rho \sum_{n=1}^{\infty} w_{n}(1) w_{n}(x) \\
& \times\left(\alpha_{1} \cos \tau \sqrt{\mu_{n}}\right. \\
& \left.+\int_{-\tau}^{0} g_{1}(\eta) \cos (\tau+\eta) \sqrt{\mu_{n}} d \eta\right), \\
& b_{2}(x)=I_{\rho} \sum_{n=1}^{\infty} \varphi_{n}(1) w_{n}(x) \\
& \times\left(\alpha_{2} \cos \tau \sqrt{\mu_{n}}\right. \\
& \left.+\int_{-\tau}^{0} g_{2}(\eta) \cos (\tau+\eta) \sqrt{\mu_{n}} d \eta\right), \\
& b_{3}(x)=\rho \sum_{n=1}^{\infty} w_{n}(1) \varphi_{n}(x) \\
& \times\left(\alpha_{1} \cos \tau \sqrt{\mu_{n}}\right. \\
& \left.+\int_{-\tau}^{0} g_{1}(\eta) \cos (\tau+\eta) \sqrt{\mu_{n}} d \eta\right), \\
& b_{4}(x)=I_{\rho} \sum_{n=1}^{\infty} \varphi_{n}(1) \varphi_{n}(x) \\
& \times\left(\alpha_{2} \cos \tau \sqrt{\mu_{n}}\right. \\
& \left.+\int_{-\tau}^{0} g_{2}(\eta) \cos (\tau+\eta) \sqrt{\mu_{n}} d \eta\right), \\
& a_{1}(x, s)=\rho \sum_{n=1}^{\infty} w_{n}(1) w_{n}(x) \\
& \times\left(\beta_{1} \frac{\sin s \sqrt{\mu_{n}}}{\sqrt{\mu_{n}}}\right. \\
& \left.-\int_{-\tau}^{s} g_{1}(\eta) \frac{\sin (\tau-s+\eta) \sqrt{\mu_{n}}}{\sqrt{\mu_{n}}} d \eta\right), \\
& a_{4}(x, s)=I_{\rho} \sum_{n=1}^{\infty} \varphi_{n}(1) \varphi_{n}(x) \\
& \times\left(\beta_{2} \frac{\sin s \sqrt{\mu_{n}}}{\sqrt{\mu_{n}}}\right. \\
& \left.-\int_{-\tau}^{s} g_{2}(\eta) \frac{\sin (\tau-s+\eta) \sqrt{\mu_{n}}}{\sqrt{\mu_{n}}} d \eta\right), \\
& b_{1}(x, s)=\rho \sum_{n=1}^{\infty} w_{n}(1) w_{n}(x) \\
& \times\left(\beta_{1} \cos s \sqrt{\mu_{n}}\right. \\
& \left.+\int_{-\tau}^{s} g_{1}(\eta) \cos (\tau-s+\eta) \sqrt{\mu_{n}} d \eta\right), \\
& b_{2}(x, s)=I_{\rho} \sum_{n=1}^{\infty} \varphi_{n}(1) w_{n}(x) \\
& \times\left(\beta_{2} \cos s \sqrt{\mu_{n}}\right. \\
& \left.+\int_{-\tau}^{s} g_{2}(\eta) \cos (\tau-s+\eta) \sqrt{\mu_{n}} d \eta\right), \\
& b_{3}(x, s)=\rho \sum_{n=1}^{\infty} w_{n}(1) \varphi_{n}(x) \\
& \times\left(\beta_{1} \cos s \sqrt{\mu_{n}}\right. \\
& \left.+\int_{-\tau}^{s} g_{1}(\eta) \cos (\tau-s+\eta) \sqrt{\mu_{n}} d \eta\right), \\
& b_{4}(x, s)=I_{\rho} \sum_{n=1}^{\infty} \varphi_{n}(1) \varphi_{n}(x) \\
& \times\left(\beta_{2} \cos s \sqrt{\mu_{n}}\right. \\
& \left.+\int_{-\tau}^{s} g_{2}(\eta) \cos (\tau-s+\eta) \sqrt{\mu_{n}} d \eta\right),
\end{aligned}
$$


and the linear operators are

$$
\begin{aligned}
& E_{1}\left(\omega_{0}, \varphi_{0}, \omega_{1}, \varphi_{1}\right)(x) \\
& =\sum_{n=1}^{\infty}\left[\cos \tau \sqrt{\mu_{n}}\left(W_{0}, \Phi_{n}\right)+\frac{\sin \tau \sqrt{\mu_{n}}}{\sqrt{\mu_{n}}}\left(W_{1}, \Phi_{n}\right)\right] w_{n}(x), \\
& E_{2}\left(\omega_{0}, \varphi_{0}, \omega_{1}, \varphi_{1}\right)(x) \\
& =\sum_{n=1}^{\infty}\left[\cos \tau \sqrt{\mu_{n}}\left(W_{0}, \Phi_{n}\right)\right. \\
& \left.+\frac{\sin \tau \sqrt{\mu_{n}}}{\sqrt{\mu_{n}}}\left(W_{1}, \Phi_{n}\right)\right] \varphi_{n}(x) \\
& E_{3}\left(\omega_{0}, \varphi_{0}, \omega_{1}, \varphi_{1}\right)(x) \\
& =\sum_{n=1}^{\infty}\left[-\sqrt{\mu_{n}} \sin \tau \sqrt{\mu_{n}}\left(W_{0}, \Phi_{n}\right)\right. \\
& \left.+\cos \tau \sqrt{\mu_{n}}\left(W_{1}, \Phi_{n}\right)\right] w_{n}(x), \\
& E_{4}\left(\omega_{0}, \varphi_{0}, \omega_{1}, \varphi_{1}\right)(x) \\
& \begin{aligned}
=\sum_{n=1}^{\infty}[ & -\sqrt{\mu_{n}} \sin \tau \sqrt{\mu_{n}}\left(W_{0}, \Phi_{n}\right) \\
& \left.+\cos \tau \sqrt{\mu_{n}}\left(W_{1}, \Phi_{n}\right)\right] \varphi_{n}(x),
\end{aligned}
\end{aligned}
$$

\section{The Proof of Theorem 1}

In this section, we will prove Theorem 1 . Here we mainly estimate the error:

$$
\begin{aligned}
& \|P(\cdot, t)-W(\cdot, t+\tau)\|_{V_{K}^{1}(0,1) \times V_{E I}^{1}(0,1)}^{2} \\
& \quad+\left\|Q(\cdot, t)-W_{t}(\cdot, t+\tau)\right\|_{L_{\rho}^{2}(0,1) \times L_{I_{\rho}}^{2}(0,1)}^{2}
\end{aligned}
$$

According to the calculation in Section 3, we have

$$
\begin{aligned}
P(x, t) & -W(x, t+\tau) \\
= & -\int_{0}^{\tau} \operatorname{Sin}((\tau-r) \mathscr{L}) B \Lambda_{1} U(t+r) d r \\
& -\int_{0}^{\tau} \operatorname{Sin}((\tau-r) \mathscr{L}) B \int_{-\tau}^{0} \Lambda_{3}(\eta) U(t+r+\eta) d \eta d r
\end{aligned}
$$

$$
\begin{aligned}
Q(x, t) & -W_{t}(x, t+\tau) \\
= & -\int_{0}^{\tau} \operatorname{Cos}((\tau-r) \mathscr{L}) B \Lambda_{1} U(t+r) d r \\
& -\int_{0}^{\tau} \operatorname{Cos}((\tau-r) \mathscr{L}) B \int_{-\tau}^{0} \Lambda_{3}(\eta) U(t+r+\eta) d \eta d r .
\end{aligned}
$$

So,

$$
\begin{aligned}
& \|P(\cdot, t)-W(\cdot, t+\tau)\|_{V_{K}^{1}(0,1) \times V_{E I}^{1}(0,1)}^{2} \\
& +\left\|Q(\cdot, t)-W_{t}(\cdot, t+\tau)\right\|_{L_{\rho}^{2}(0,1) \times L_{I_{\rho}}^{2}(0,1)}^{2} \\
& \leq 4\left(\alpha_{1} \rho\right)^{2} \sum_{n=1}^{\infty}\left|\omega_{n}(1)\right|^{2} \\
& \times\left|\int_{0}^{\tau} \sin \sqrt{\mu_{n}}(\tau-r) u_{1}(r+t) d r\right|^{2} \\
& +4\left(\alpha_{2} I_{\rho}\right)^{2} \sum_{n=1}^{\infty}\left|\varphi_{n}(1)\right|^{2} \\
& \times\left|\int_{0}^{\tau} \sin \sqrt{\mu_{n}}(\tau-r) u_{2}(r+t) d r\right|^{2} \\
& +4 \rho^{2} \sum_{n=1}^{\infty}\left|\omega_{n}(1)\right|^{2} \\
& \times\left|\int_{0}^{\tau} \sin \sqrt{\mu_{n}}(\tau-r)\left(\int_{-\tau}^{0} g_{1}(\eta) u_{1}(r+t+\eta) d \eta\right) d r\right|^{2} \\
& +4 I_{\rho}^{2} \sum_{n=1}^{\infty}\left|\varphi_{n}(1)\right|^{2} \\
& \times\left|\int_{0}^{\tau} \sin \sqrt{\mu_{n}}(\tau-r)\left(\int_{-\tau}^{0} g_{2}(\eta) u_{2}(r+t+\eta) d \eta\right) d r\right|^{2} \\
& +4\left(\alpha_{1} \rho\right)^{2} \sum_{n=1}^{\infty}\left|\omega_{n}(1)\right|^{2} \\
& \times\left|\int_{0}^{\tau} \cos \sqrt{\mu_{n}}(\tau-r) u_{1}(r+t) d r\right|^{2} \\
& +4\left(\alpha_{2} I_{\rho}\right)^{2} \sum_{n=1}^{\infty}\left|\varphi_{n}(1)\right|^{2} \\
& \times\left|\int_{0}^{\tau} \cos \sqrt{\mu_{n}}(\tau-r) u_{2}(r+t) d r\right|^{2} \\
& +4 \rho^{2} \sum_{n=1}^{\infty}\left|\omega_{n}(1)\right|^{2} \\
& \times\left|\int_{0}^{\tau} \cos \sqrt{\mu_{n}}(\tau-r)\left(\int_{-\tau}^{0} g_{1}(\eta) u_{1}(r+t+\eta) d \eta\right) d r\right|^{2} \\
& +4 I_{\rho}^{2} \sum_{n=1}^{\infty}\left|\varphi_{n}(1)\right|^{2} \\
& \times\left|\int_{0}^{\tau} \cos \sqrt{\mu_{n}}(\tau-r)\left(\int_{-\tau}^{0} g_{2}(\eta) u_{2}(r+t+\eta) d \eta\right) d r\right|^{2} .
\end{aligned}
$$


Note that $\left\{\cos \sqrt{\mu_{n}} t, \sin \sqrt{\mu_{n}} t ; n \in \mathbb{N}\right\}$ is a Riesz basis sequence for $L^{2}[0, \tau]$. Thus, there exist positive constants $M_{i}(i=1,2,3,4)$ such that

$$
\begin{aligned}
\| P(\cdot, t) & -W(\cdot, t+\tau) \|_{V_{K}^{1}(0,1) \times V_{E I}^{1}(0,1)}^{2} \\
& +\left\|Q(\cdot, t)-W_{t}(\cdot, t+\tau)\right\|_{L_{\rho}^{2}(0,1) \times L_{I_{\rho}}^{2}(0,1)}^{2} \\
\leq & M_{1}{ }^{2} \int_{0}^{\tau}\left|u_{1}(r+t)\right|^{2} d r+M_{2}{ }^{2} \int_{0}^{\tau}\left|u_{2}(r+t)\right|^{2} d r \\
& +M_{3}{ }^{2} \int_{0}^{\tau}\left|\int_{-\tau}^{0} g_{1}(\eta) u_{1}(t+r+\eta) d \eta\right|^{2} d r \\
& +M_{4}{ }^{2} \int_{0}^{\tau}\left|\int_{-\tau}^{0} g_{2}(\eta) u_{2}(t+r+\eta) d \eta\right|^{2} d r \\
\leq & M_{1}{ }^{2} \int_{0}^{\tau}\left|u_{1}(r+t)\right|^{2} d r+M_{2}{ }^{2} \int_{0}^{\tau}\left|u_{2}(r+t)\right|^{2} d r \\
& +M_{3}{ }^{2} \tau^{2} \int_{-\tau}^{0}\left|g_{1}(\eta)\right|^{2} d \eta \int_{t-\tau}^{t+\tau}\left|u_{1}(s)\right|^{2} d s \\
& +M_{4}{ }^{2} \tau^{2} \int_{-\tau}^{0}\left|g_{2}(\eta)\right|^{2} d \eta \int_{t-\tau}^{t+\tau}\left|u_{2}(s)\right|^{2} d s .
\end{aligned}
$$

Let $(P(x, t), Q(x, t))$ be the solution to (11), and $E(t)$ be its energy functional; then we have

$$
\begin{gathered}
E(t)=\frac{1}{2}\|(P(x, t), Q(x, t))\|_{\mathscr{H}}^{2}, \\
\frac{d E(t)}{d t}=-U_{1}^{2}(P, Q)(t)-U_{2}^{2}(P, Q)(t) .
\end{gathered}
$$

Therefore, we have

$$
\begin{aligned}
& \int_{0}^{\tau}\|U(t+r)\|_{\mathbb{R}^{2}}^{2} d r \\
& \quad=\int_{t}^{t+\tau}\left(U_{1}^{2}(P, Q)(r)+U_{2}^{2}(P, Q)(r)\right) d r \\
& \quad=E(t)-E(t+\tau), \\
& \int_{0}^{\tau}\|U(t-r)\|_{\mathbb{R}^{2}}^{2} d r=E(t-\tau)-E(t) .
\end{aligned}
$$

So, we can get

$$
\begin{aligned}
& \|P(\cdot, t)-W(\cdot, t+\tau)\|_{V_{K}^{1}(0,1) \times V_{E I}^{1}(0,1)}^{2} \\
& +\left\|Q(\cdot, t)-W_{t}(\cdot, t+\tau)\right\|_{L_{\rho}^{2}(0,1) \times L_{I_{\rho}}^{2}(0,1)}^{2} \\
& \leq \max _{i=1,2}\left\{M_{i}^{2}\right\}[E(t)-E(t+\tau)] \\
& \quad+\max _{j=3,4}\left\{M_{j}^{2}\right\} \tau^{2} \int_{-\tau}^{0}\left(\left|g_{1}(\eta)\right|^{2}+\left|g_{2}(\eta)\right|^{2}\right) d \eta \\
& \quad \times[E(t-\tau)-E(t+\tau)] .
\end{aligned}
$$

If $(P(x, t), Q(x, t))$ is exponential stable, there exists a positive constant $\varepsilon>0$ such that $E(t) \leq E(0) e^{-\varepsilon t}$. We can obtain the following result from above:

$$
\begin{aligned}
& \|P(\cdot, t)-W(\cdot, t+\tau)\|_{V_{K}^{1}(0,1) \times V_{E I}^{1}(0,1)}^{2} \\
& \quad+\left\|Q(\cdot, t)-W_{t}(\cdot, t+\tau)\right\|_{L_{\rho}^{2}(0,1) \times L_{I_{\rho}}^{2}(0,1)}^{2} \leq M e^{-\varepsilon(t-\tau)},
\end{aligned}
$$

where $M$ is a positive constant. So $\left(W(x, t), W_{t}(x, t)\right)$ also decays exponentially.

\section{The Proof of Theorem 2}

In this section, we will discuss the stability of system (11). At first we consider $L^{2}$ well posed of the system (6). For the sake of simplicity, we use the vector form of (6); that is,

$$
\begin{aligned}
& P_{t}(x, t)= Q(x, t)+\operatorname{Sin}(\tau \mathscr{L}) B \Lambda_{1} U(t) \\
&+\int_{-\tau}^{0} \operatorname{Sin}((\tau+\eta) \mathscr{L}) B \Lambda_{3}(\eta) U(t) d \eta, \\
& Q_{t}(x, t)=- \mathscr{L} P(x, t)+\operatorname{Cos}(\tau \mathscr{L}) B \Lambda_{1} U(t) \\
&+\int_{-\tau}^{0} \operatorname{Cos}((\tau+\eta) \mathscr{L}) B \Lambda_{3}(\eta) U(t) d \eta, \\
& \Gamma_{N} P(\cdot, t)=\Lambda_{2} U(t), \\
& P(0, t)=Q(0, t)=0, \\
& P(x, 0)=P_{0}(x), \quad Q(x, 0)=Q_{0}(x) .
\end{aligned}
$$

The observation system corresponding to (55) is

$$
\begin{gathered}
W_{t}(x, t)=V(x, t), \quad x \in(0,1), t>0, \\
V_{t}(x, t)=-\mathscr{L} W(x, t), \quad x \in(0,1), t>0, \\
W(x, 0)=W_{0}, \\
V(x, 0)=V_{0}, \\
y_{1}(t)=\beta_{1} z(1, t)+\int_{0}^{1} K\left(w_{x}(x, t)-\varphi(x, t)\right) \\
\quad \times\left(a_{1}^{\prime}(x)-a_{3}(x)\right) d x \\
+\int_{0}^{1} E I \varphi_{x}(x, t) a_{3}^{\prime}(x) d x \\
+\int_{0}^{1} \rho z(x, t) b_{1}(x) d x+\int_{0}^{1} I_{\rho} \psi(x, t) b_{3}(x) d x \\
y_{2}(t)=\beta_{2} \psi(1, t)+\int_{0}^{1} K\left(w_{x}(x, t)-\varphi(x, t)\right) \\
+\int_{0}^{1} E I \varphi_{x}(x, t) a_{4}^{\prime}(x) d x \\
\times\left(a_{2}^{\prime}(x)-a_{4}(x)\right) d x
\end{gathered}
$$


where $W(x, t)=(w(x, t), \varphi(x, t))^{T}$ and $V(x, t)=(z(x, t)$, $\psi(x, t))^{T}$.

We can write the observation as

$$
\begin{aligned}
y_{1}(t)= & \beta_{1} z(1, t)+\left\langle W(\cdot, t),\left(a_{1}, a_{3}\right)^{T}\right\rangle_{V_{k}^{1} \times V_{E I}^{1}} \\
& +\left\langle V(\cdot, t),\left(b_{1}, b_{3}\right)^{T}\right\rangle_{L_{\rho}^{2} \times L_{I_{\rho}}^{2}}, \\
y_{2}(t)= & \beta_{1} \psi(1, t)+\left\langle W(\cdot, t),\left(a_{2}, a_{4}\right)^{T}\right\rangle_{V_{k}^{1} \times V_{E I}^{1}} \\
& +\left\langle V(\cdot, t),\left(b_{2}, b_{4}\right)^{T}\right\rangle_{L_{\rho}^{2} \times L_{I_{\rho}}^{2}} .
\end{aligned}
$$

Since

$$
\begin{aligned}
\left(\begin{array}{ll}
a_{1}(x) & a_{2}(x) \\
a_{3}(x) & a_{4}(x)
\end{array}\right) & \\
= & \operatorname{Sin}(\tau \mathscr{L}) B \Lambda_{1} \\
& \quad+\int_{-\tau}^{0} \operatorname{Sin}((\tau+\eta) \mathscr{L}) B \Lambda_{3}(\eta) d \eta,
\end{aligned}
$$

$$
\begin{aligned}
& \left(\begin{array}{ll}
b_{1}(x) & b_{2}(x) \\
b_{3}(x) & b_{4}(x)
\end{array}\right) \\
& =\operatorname{Cos}(\tau \mathscr{L}) B \Lambda_{1} \\
& \quad+\int_{-\tau}^{0} \operatorname{Cos}((\tau+\eta) \mathscr{L}) B \Lambda_{3}(\eta) d \eta,
\end{aligned}
$$

$Y(t)=C(W, V)=\Lambda_{2} V(1, t)$

$$
\begin{aligned}
& +\left[\Lambda_{1} \mathscr{L}^{1 / 2} \operatorname{Sin}(\tau \mathscr{L}) B\right. \\
& \left.+\mathscr{L}^{1 / 2} \int_{-\tau}^{0} \operatorname{Sin}((\tau+\eta) \mathscr{L}) B \Lambda_{3}(\eta) d \eta\right]^{*} \mathscr{L}^{1 / 2} W(t) \\
& +\left[\Lambda_{1} \operatorname{Cos}(\tau \mathscr{L}) B\right. \\
& \left.+\int_{-\tau}^{0} \operatorname{Cos}((\tau+\eta) \mathscr{L}) B \Lambda_{3}(\eta) d \eta\right]^{*} V(t) .
\end{aligned}
$$

Taking the Laplace transform for above equation leads to, for any $\mathfrak{R} \lambda>0$,

$$
\begin{aligned}
\lambda P(x)= & Q(x)+\operatorname{Sin}(\tau \mathscr{L}) B \Lambda_{1} U(\lambda) \\
& +\int_{-\tau}^{0} \operatorname{Sin}((\tau+\eta) \mathscr{L}) B \Lambda_{3}(\eta) U(\lambda) d \eta, \\
x \in(0,1), & \\
\lambda Q(x)=- & \mathscr{L} P(x)+\operatorname{Cos}(\tau \mathscr{L}) B \Lambda_{1} U(\lambda) \\
+ & \int_{-\tau}^{0} \operatorname{Cos}((\tau+\eta) \mathscr{L}) B \Lambda_{3}(\eta) U(\lambda) d \eta, \\
& \Gamma_{N} P(\cdot, t)=\Lambda_{2} U(\lambda), \\
& P(0, t)=Q(0, t)=0,
\end{aligned}
$$

$$
\begin{aligned}
Y(\lambda)=\Lambda_{2} Q(1) \\
+\left[\Lambda_{1} \mathscr{L}^{1 / 2} \operatorname{Sin}(\tau \mathscr{L}) B\right. \\
\left.+\mathscr{L}^{1 / 2} \int_{-\tau}^{0} \operatorname{Sin}((\tau+\eta) \mathscr{L}) B \Lambda_{3}(\eta) d \eta\right]^{*} \mathscr{L}^{1 / 2} P(\cdot) \\
+\left[\Lambda_{1} \operatorname{Cos}(\tau \mathscr{L}) B\right. \\
\left.\quad+\int_{-\tau}^{0} \operatorname{Cos}((\tau+\eta) \mathscr{L}) B \Lambda_{3}(\eta) d \eta\right]^{*} Q(\cdot) .
\end{aligned}
$$

We have the following results by solving (59):

$$
\begin{aligned}
\left(\lambda^{2}+\mathscr{L}\right) p(x) & \\
= & {\left[\lambda \left(\operatorname{Sin}(\tau \mathscr{L}) B \Lambda_{1}\right.\right.} \\
& \left.\quad+\int_{-\tau}^{0} \operatorname{Sin}((\tau+\eta) \mathscr{L}) B \Lambda_{3}(\eta) d \eta\right) \\
& +\operatorname{Cos}(\tau \mathscr{L}) B \Lambda_{1} \\
& +\int_{-\tau}^{0} \operatorname{Cos}((\tau+\eta) \mathscr{L}) B \Lambda_{3}(\eta) d \eta \\
& \left.+B \Lambda_{2}\right] U(\lambda), \\
Y(\lambda)=\left[\begin{array}{l}
\lambda \\
\quad
\end{array}\right. & +\int_{-\tau}^{0} \operatorname{Sin}(\tau \mathscr{L}) B \Lambda_{1} \\
+ & \operatorname{Cos}(\tau \mathscr{L}) B \Lambda_{1}+\int_{-\tau}^{0} \operatorname{Cos}((\tau+\eta) \mathscr{L}) B \Lambda_{3}(\eta) d \eta \\
+ & \left.B \Lambda_{2}\right]^{*} P .
\end{aligned}
$$

So we can get

$$
\begin{aligned}
Y(\lambda)=[ & \lambda\left(\sin (\tau \mathscr{L}) B \Lambda_{1}\right. \\
& \left.+\int_{-\tau}^{0} \operatorname{Sin}((\tau+\eta) \mathscr{L}) B \Lambda_{3}(\eta) d \eta\right) \\
& +\operatorname{Cos}(\tau \mathscr{L}) B \Lambda_{1}+\int_{-\tau}^{0} \operatorname{Cos}((\tau+\eta) \mathscr{L}) B \Lambda_{3}(\eta) d \eta \\
& \left.+B \Lambda_{2}\right]^{*}\left(\lambda^{2}+\mathscr{L}\right)^{-1}
\end{aligned}
$$




$$
\begin{aligned}
\times[ & \lambda\left(\operatorname{Sin}(\tau \mathscr{L}) B \Lambda_{1}\right. \\
& \left.\quad+\int_{-\tau}^{0} \operatorname{Sin}((\tau+\eta) \mathscr{L}) B \Lambda_{3}(\eta) d \eta\right) \\
& +\operatorname{Cos}(\tau \mathscr{L}) B \Lambda_{1} \\
& \left.+\int_{-\tau}^{0} \operatorname{Cos}((\tau+\eta) \mathscr{L}) B \Lambda_{3}(\eta) d \eta+B \Lambda_{2}\right] U(\lambda),
\end{aligned}
$$

and hence the transform matrix is

$$
\begin{aligned}
H(\lambda)=[ & \lambda\left(\operatorname{Sin}(\tau \mathscr{L}) B \Lambda_{1}\right. \\
& \left.+\int_{-\tau}^{0} \operatorname{Sin}((\tau+\eta) \mathscr{L}) B \Lambda_{3}(\eta) d \eta\right) \\
& +\operatorname{Cos}(\tau \mathscr{L}) B \Lambda_{1} \\
+ & \int_{-\tau}^{0} \operatorname{Cos}((\tau+\eta) \mathscr{L}) B \Lambda_{3}(\eta) d \eta \\
+ & \left.B \Lambda_{2}\right]^{*}\left(\lambda^{2}+\mathscr{L}\right)^{-1} \\
\times & \lambda\left(\operatorname{Sin}(\tau \mathscr{L}) B \Lambda_{1}\right. \\
& \left.+\int_{-\tau}^{0} \operatorname{Sin}((\tau+\eta) \mathscr{L}) B \Lambda_{3}(\eta) d \eta\right) \\
& +\operatorname{Cos}(\tau \mathscr{L}) B \Lambda_{1} \\
& \left.+\int_{-\tau}^{0} \operatorname{Cos}((\tau+\eta) \mathscr{L}) B \Lambda_{3}(\eta) d \eta+B \Lambda_{2}\right] .
\end{aligned}
$$

For any $Z=\left(z_{1}, z_{2}\right) \in \mathbb{C}^{2}$, we can get

$$
\begin{aligned}
& (H(\lambda) Z, Z)_{\mathbb{C}^{2}} \\
& =\sum_{n=1}^{\infty} \frac{1}{\lambda^{2}+\mu_{n}} \\
& \times \mid\left(\mid \lambda\left(\operatorname{Sin}(\tau \mathscr{L}) B \Lambda_{1}\right.\right. \\
& \left.\quad+\int_{-\tau}^{0} \operatorname{Sin}((\tau+\eta) \mathscr{L}) B \Lambda_{3}(\eta) d \eta\right) \\
& \quad+\operatorname{Cos}(\tau \mathscr{L}) B \Lambda_{1}+\int_{-\tau}^{0} \operatorname{Cos}((\tau+\eta) \mathscr{L}) \\
& \left.\left.\quad \times B \Lambda_{3}(\eta) d \eta+B \Lambda_{2}\right] Z, \Phi_{n}\right)\left._{L_{\rho}^{2} \times L_{I_{\rho}}^{2}}\right|^{2}
\end{aligned}
$$

We can easily get

$$
\begin{gathered}
\left(B \Lambda_{2} Z, \Phi_{n}\right)_{L_{\rho}^{2} \times L_{I_{\rho}}^{2}}=\beta_{1} z_{1} \rho w_{n}(1)+\beta_{2} z_{2} I_{\rho} \varphi_{n}(1), \\
\left(\operatorname{Sin}(\tau \mathscr{L}) B \Lambda_{1} Z, \Phi_{n}\right)_{L_{\rho}^{2} \times L_{I_{\rho}}^{2}} \\
=\frac{\operatorname{Sin}\left(\tau \sqrt{\mu_{n}}\right)}{\sqrt{\mu_{n}}}\left[\alpha_{1} z_{1} \rho w_{n}(1)+\alpha_{2} z_{2} I_{\rho} \varphi_{n}(1)\right], \\
\left(\operatorname{Cos}(\tau \mathscr{L}) B \Lambda_{1} Z, \Phi_{n}\right)_{L_{\rho}^{2} \times L_{I_{\rho}}^{2}} \\
=\operatorname{Cos}\left(\tau \sqrt{\mu_{n}}\right)\left[\alpha_{1} z_{1} \rho w_{n}(1)+\alpha_{2} z_{2} I_{\rho} \varphi_{n}(1)\right], \\
\left(\int_{-\tau}^{0} \operatorname{Sin}(\tau+\eta) \mathscr{L} B \Lambda_{3}(\eta) d \eta Z, \Phi_{n}\right)_{L_{\rho}^{2} \times L_{I_{\rho}}^{2}} \\
=\int_{-\tau}^{0} \frac{\operatorname{Sin}\left((\tau+\eta) \sqrt{\mu_{n}}\right)}{\sqrt{\mu_{n}}} \\
\quad \times\left[g_{1}(\eta) z_{1} \rho w_{n}(1)+g_{2}(\eta) z_{2} I_{\rho} \varphi_{n}(1)\right] d \eta \\
\left(\int_{-\tau}^{0} \operatorname{Cos}(\tau+\eta) \mathscr{L} B \Lambda_{3}(\eta) d \eta Z, \Phi_{n}\right)_{L_{\rho}^{2} \times L_{I_{\rho}}^{2}} \\
\quad=\int_{-\tau}^{0} \operatorname{Cos}\left((\tau+\eta) \sqrt{\mu_{n}}\right) \\
\times\left[g_{1}(\eta) z_{1} \rho w_{n}(1)+g_{2}(\eta) z_{2} I_{\rho} \varphi_{n}(1)\right] d \eta .
\end{gathered}
$$

Thus, we have

$$
\begin{aligned}
& \mid\left(\left[\lambda\left(\operatorname{Sin}(\tau \mathscr{L}) B \Lambda_{1}+\int_{-\tau}^{0} \operatorname{Sin}((\tau+\eta) \mathscr{L}) B \Lambda_{3}(\eta) d \eta\right)\right.\right. \\
& \quad+\operatorname{Cos}(\tau \mathscr{L}) B \Lambda_{1}+\int_{-\tau}^{0} \operatorname{Cos}((\tau+\eta) \mathscr{L}) B \Lambda_{3}(\eta) d \eta \\
& \left.\left.\quad+B \Lambda_{2}\right] Z, \Phi_{n}\right)\left._{L_{\rho}^{2} \times L_{I_{\rho}}^{2}}\right|^{2} \\
& \leq F\left|\frac{|\lambda|^{2}}{\mu_{n}}+2\right|\left[\rho\left|\omega_{n}(1)\right|^{2}+I_{\rho}\left|\varphi_{n}(1)\right|^{2}\right]\|Z\|_{\mathbb{C}^{2}}^{2},
\end{aligned}
$$

where $F$ is a positive constant dependent on $\alpha_{i}, \beta_{i}, g_{i}(\eta), i=$ 1,2 . Therefore, we have the following result:

$$
\begin{aligned}
\|H(\lambda)\| \leq & F \sum_{n=1}^{\infty} \frac{1}{|\lambda|^{2}+\mu_{n}}\left|\frac{|\lambda|^{2}}{\mu_{n}}+2\right| \\
& \times\left[\rho\left|\omega_{n}(1)\right|^{2}+I_{\rho}\left|\varphi_{n}(1)\right|^{2}\right] .
\end{aligned}
$$

From Lemma 4, we have

$$
\sup _{\mathfrak{R} \lambda>\delta>0}\|H(\lambda)\|<\infty .
$$

Hence the system (6) is $L_{\text {loc }}^{2}$ well posed (see, [15]).

Next, we consider the exact observability of the system (6). 
Lemma 6 (see [16]). Let $\mathscr{H}$ be a separable Hilbert space, and let $\mathscr{L}$ be a unbounded positive definite operator. Assume that $\mathscr{L}$ satisfies the following conditions:

(1) $\mathscr{L}$ has compact resolvent and its spectrum is $\sigma(\mathscr{L})=$ $\left\{\mu_{n} ; n \in \mathbb{N}\right\}$

(2) the spectra of $\mathscr{L}$ satisfy the separable condition

$$
\inf _{n \neq m}\left|\sqrt{\mu_{n}}-\sqrt{\mu_{m}}\right|=\delta>0 ;
$$

(3) the corresponding eigenvectors $\left\{\Phi_{n} ; n \in \mathbb{N}\right\}$ with $\left\|\Phi_{n}\right\|_{\mathscr{H}}=1$ form a normalized orthogonal basis for $\mathscr{H}$.

Let $\mathbb{Y}$ be a Hilbert space. Assume that $C: D(\mathscr{L}) \rightarrow \mathbb{Y}$ is an admissible observation operator for $\mathscr{L}$. Then the following system:

$$
\begin{aligned}
& Z_{t t}+\mathscr{L} Z(t)=0, \quad Z(0)=Z_{0}, \\
& Z_{t}(0)=Z_{1}, \quad Y(t)=C\left(Z, Z_{t}\right)
\end{aligned}
$$

is exactly observable in finite time in the energy space $D\left(\mathscr{L}^{1 / 2}\right) \times \mathscr{H}$ if and only if

$$
\inf _{n \in \mathbb{N}}\left\|C\left(\frac{\Phi_{n}}{i \sqrt{\mu_{n}}}, \Phi_{n}\right)\right\|_{\mathbb{Y}}>0 .
$$

Now we apply Lemma 6 to the system (55). We can easily know that the condition (68) is fulfilled when $c_{1}=$ $\sqrt{K / \rho} \neq c_{2}=\sqrt{E I / I_{\rho}}($ see Remark 2.1 in [8]).

For $\Phi_{n}(x)=\left(w_{n}(x), \varphi_{n}(x)\right)^{T}$, we have

$$
\begin{aligned}
C\left(\frac{\Phi_{n}}{i \sqrt{\mu_{n}}}, \Phi_{n}\right) \\
=\Lambda_{2} \Phi_{n}(1) \\
+\left[\Lambda_{1} \mathscr{L}^{1 / 2} \operatorname{Sin}(\tau \mathscr{L}) B\right. \\
\left.+\mathscr{L}^{1 / 2} \int_{-\tau}^{0} \operatorname{Sin}((\tau+\eta) \mathscr{L}) B \Lambda_{3}(\eta) d \eta\right]^{*} \mathscr{L}^{1 / 2} \frac{\Phi_{n}}{i \sqrt{\mu_{n}}} \\
+\left[\Lambda_{1} \operatorname{Cos}(\tau \mathscr{L}) B\right. \\
\left.+\int_{-\tau}^{0} \operatorname{Cos}((\tau+\eta) \mathscr{L}) B \Lambda_{3}(\eta) d \eta\right]^{*} \Phi_{n} .
\end{aligned}
$$

For any $Z=\left(z_{1}, z_{2}\right) \in \mathbb{C}^{2}$, we have

$$
\begin{aligned}
\left(\Lambda_{1}\right. & \left.\left(\mathscr{L}^{1 / 2} \operatorname{Sin}(\tau \mathscr{L}) B\right)^{*} \mathscr{L}^{1 / 2} \frac{\Phi_{n}}{i \sqrt{\mu_{n}}}, Z\right)_{\mathbb{C}^{2}} \\
& =\left(\mathscr{L}^{1 / 2} \frac{\Phi_{n}}{i \sqrt{\mu_{n}}}, \mathscr{L}^{1 / 2} \operatorname{Sin}(\tau \mathscr{L}) B \Lambda_{1} Z\right)_{L_{\rho}^{2} \times L_{I_{\rho}}^{2}} \\
& =-i \sqrt{\mu_{n}}\left(\Phi_{n}, \operatorname{Sin}(\tau \mathscr{L}) B \Lambda_{1} Z\right)_{L_{\rho}^{2} \times L_{I_{\rho}}^{2}} \\
& =-i \sin \left(\sqrt{\mu_{n}} \tau\right) \overline{\left[\alpha_{1} z_{1} \rho \omega_{n}(1)+\alpha_{2} z_{2} I_{\rho} \varphi_{n}(1)\right] .}
\end{aligned}
$$

Similarity, we have

$$
\begin{gathered}
\left(\left(\mathscr{L}^{1 / 2} \int_{-\tau}^{0} \operatorname{Sin}((\tau+\eta) \mathscr{L}) B \Lambda_{3}(\eta) d \eta\right)^{*} \mathscr{L}^{1 / 2} \frac{\Phi_{n}}{i \sqrt{\mu_{n}}}, Z\right)_{\mathbb{C}^{2}} \\
=-i \int_{-\tau}^{0} \sin \left((\tau+\eta) \sqrt{\mu_{n}}\right) \\
\quad \times \overline{\left[g_{1}(\eta) z_{1} \rho \omega_{n}(1)+g_{2}(\eta) z_{2} I_{\rho} \varphi_{n}(1)\right]} d \eta, \\
\left(\Lambda_{1}(\operatorname{Cos}(\tau \mathscr{L}) B)^{*} \Phi_{n}, Z\right)_{\mathbb{C}^{2}} \\
=\cos \left(\sqrt{\mu_{n}} \tau\right) \overline{\left[\alpha_{1} z_{1} \rho \omega_{n}(1)+\alpha_{2} z_{2} I_{\rho} \varphi_{n}(1)\right]}, \\
\left(\left(\int_{-\tau}^{0} \operatorname{Cos}((\tau+\eta) \mathscr{L}) B \Lambda_{3}(\eta) d \eta\right)^{*} \Phi_{n}, Z\right)_{\mathbb{C}^{2}} \\
=\int_{-\tau}^{0} \cos \left((\tau+\eta) \sqrt{\mu_{n}}\right) \\
\times\left[\overline{\left[g_{1}(\eta) z_{1} \rho \omega_{n}(1)+g_{2}(\eta) z_{2} I_{\rho} \varphi_{n}(1)\right]} d \eta .\right.
\end{gathered}
$$

Thus it holds that

$$
\begin{aligned}
& C\left(\frac{\Phi_{n}}{i \sqrt{\mu_{n}}}, \Phi_{n}\right) \\
& =\left(\begin{array}{l}
\left(\frac{\beta_{1}}{\rho}+\alpha_{1} e^{-i \tau \sqrt{\mu_{n}}}+\int_{-\tau}^{0} g_{1}(\eta) e^{-i\left((\tau+\eta) \sqrt{\mu_{n}}\right)} d \eta\right) \rho w_{n}(1) \\
\left(\frac{\beta_{2}}{I_{\rho}}+\alpha_{2} e^{-i \tau \sqrt{\mu_{n}}}+\int_{-\tau}^{0} g_{2}(\eta) e^{-i\left((\tau+\eta) \sqrt{\mu_{n}}\right)} d \eta\right) I_{\rho} \varphi_{n}(1)
\end{array}\right) .
\end{aligned}
$$

Set

$$
\begin{aligned}
& \xi_{n}^{(1)}=\int_{-\tau}^{0} g_{1}(\eta) e^{-i \sqrt{\mu_{n}}(\tau+\eta)} d \eta, \\
& \xi_{n}^{(2)}=\int_{-\tau}^{0} g_{2}(\eta) e^{-i \sqrt{\mu_{n}}(\tau+\eta)} d \eta .
\end{aligned}
$$

Then

$$
\begin{aligned}
\left\|C\left(\frac{\Phi_{n}}{i \sqrt{\mu_{n}}}, \Phi_{n}\right)\right\|_{\mathbb{C}^{2}}^{2}= & \left|\frac{\beta_{1}}{\rho}+\alpha_{1} e^{-i \tau \sqrt{\mu_{n}}}+\xi_{n}^{(1)}\right|^{2}\left|\rho w_{n}(1)\right|^{2} \\
& +\left|\frac{\beta_{2}}{I_{\rho}}+\alpha_{2} e^{-i \tau \sqrt{\mu_{n}}}+\xi_{n}^{(2)}\right|^{2}\left|I_{\rho} \varphi_{n}(1)\right|^{2} .
\end{aligned}
$$

Obviously, when

$$
\begin{aligned}
& A_{1}=\inf _{n}\left|\frac{\beta_{1}}{\rho}+\alpha_{1} e^{-i \tau \sqrt{\mu_{n}}}+\xi_{n}^{(1)}\right|>0, \\
& A_{2}=\inf _{n}\left|\frac{\beta_{2}}{I_{\rho}}+\alpha_{2} e^{-i \tau \sqrt{\mu_{n}}}+\xi_{n}^{(2)}\right|>0,
\end{aligned}
$$


we have

$$
\begin{aligned}
& \left\|C\left(\frac{\Phi_{n}}{i \sqrt{\mu_{n}}}, \Phi_{n}\right)\right\|_{\mathbb{C}^{2}}^{2} \\
& \quad \geq A_{1}^{2}\left|\rho w_{n}(1)\right|^{2}+A_{2}^{2}\left|I_{\rho} \varphi_{n}(1)\right|^{2} \\
& \quad \geq \min \left\{A_{1}, A_{2}\right\}\left(\left|\rho w_{n}(1)\right|^{2}+\left|I_{\rho} \varphi_{n}(1)\right|^{2}\right) ;
\end{aligned}
$$

using Lemma 4,

$$
\inf _{n}\left\|C\left(\frac{\Phi_{n}}{i \sqrt{\mu_{n}}}, \Phi_{n}\right)\right\|_{\mathbb{C}^{2}}^{2}>0 .
$$

According to Lemma 6, the system (56) is exactly observable in finite time, and hence the closed-loop system (11) is exponentially stable.

If for all $n \in \mathbb{N}$,

$$
\begin{aligned}
& \left|\frac{\beta_{1}}{\rho}+\alpha_{1} e^{-i \tau \sqrt{\mu_{n}}}+\xi_{n}^{(1)}\right|>0, \\
& \left|\frac{\beta_{2}}{I_{\rho}}+\alpha_{2} e^{-i \tau \sqrt{\mu_{n}}}+\xi_{n}^{(2)}\right|>0,
\end{aligned}
$$

we can see that in this case, there is no eigenvalue of system (11) on the imaginary axis. Moreover, if the conditions

$$
\begin{aligned}
& \inf _{n}\left|\frac{\beta_{1}}{\rho}+\alpha_{1} e^{-i \tau \sqrt{\mu_{n}}}+\xi_{n}^{(1)}\right|=0, \\
& \inf _{n}\left|\frac{\beta_{2}}{I_{\rho}}+\alpha_{2} e^{-i \tau \sqrt{\mu_{n}}}+\xi_{n}^{(2)}\right|=0
\end{aligned}
$$

hold, then the imaginary axis is an asymptote of the eigenvalues of the system (11). Therefore, the stability theorem [17] asserts then that the system (11) is asymptotically stable. Therefore, we get the result of the Theorem 2 .

\section{Conclusion}

In this paper, we designed a new controller for a Timoshenko beam with distributed delay in the boundary that stabilizes exponentially the system. In the design process of new controllers, there are main steps: (1) to translate the delay system into a system without delay; (2) for the undelay system, we used the collocated feedback law to obtain the control signals; (3) using the obtained control signals, act on the delay system. This control strategy can be regarded as extension form of [15]. In the stability analysis, the key trick is to use the exact observability of the dual system in finite time to obtain the exponential stability of the closed-loop system.

In the proof of main result, the condition $K / \rho \neq E I / I_{\rho}$ is used to ensure the separability of the spectrum (see, the condition (2)) in Lemma 6). In the statement of our result
(Theorem 2), the conditions are stronger than the practice; in fact,

$$
\begin{aligned}
\left\|C\left(\frac{\Phi_{n}}{i \sqrt{\mu_{n}}}, \Phi_{n}\right)\right\|_{\mathbb{C}^{2}}^{2}= & \left|\frac{\beta_{1}}{\rho}+\alpha_{1} e^{-i \tau \sqrt{\mu_{n}}}+\xi_{n}^{(1)}\right|^{2}\left|\rho w_{n}(1)\right|^{2} \\
& +\left|\frac{\beta_{2}}{I_{\rho}}+\alpha_{2} e^{-i \tau \sqrt{\mu_{n}}}+\xi_{n}^{(2)}\right|^{2}\left|I_{\rho} \varphi_{n}(1)\right|^{2} ;
\end{aligned}
$$

one only needs to request

$$
\inf _{n}\left\|C\left(\frac{\Phi_{n}}{i \sqrt{\mu_{n}}}, \Phi_{n}\right)\right\|_{\mathbb{C}^{2}}^{2}>0,
$$

so, the conditions

$$
\begin{aligned}
& \inf _{n}\left|\frac{\beta_{1}}{\rho}+\alpha_{1} e^{-i \tau \sqrt{\mu_{n}}}+\xi_{n}^{(1)}\right|>0, \\
& \inf _{n}\left|\frac{\beta_{2}}{I_{\rho}}+\alpha_{2} e^{-i \tau \sqrt{\mu_{n}}}+\xi_{n}^{(2)}\right|>0,
\end{aligned}
$$

are sufficient, but not necessary. Since

$$
\begin{aligned}
& \xi_{n}^{(1)}=\int_{-\tau}^{0} g_{1}(\eta) e^{i \sqrt{\mu_{n}}(\tau-\eta)} d \eta, \\
& \xi_{n}^{(2)}=\int_{-\tau}^{0} g_{2}(\eta) e^{i \sqrt{\mu_{n}}(\tau-\eta)} d \eta,
\end{aligned}
$$

so $\lim _{n \rightarrow \infty} \xi_{n}^{(j)}=0, j=1,2$. Therefore, when

$$
\begin{aligned}
& \left|\frac{\beta_{1}}{\rho}+\alpha_{1} e^{-i \tau \sqrt{\mu_{n}}}+\xi_{n}^{(1)}\right|>0, \\
& \left|\frac{\beta_{2}}{I_{\rho}}+\alpha_{2} e^{-i \tau \sqrt{\mu_{n}}}+\xi_{n}^{(2)}\right|>0
\end{aligned}
$$

that means that there is no eigenvalue on the imaginary axis, and

$$
\begin{aligned}
& \inf _{n}\left|\frac{\beta_{1}}{\rho}+\alpha_{1} e^{-i \tau \sqrt{\mu_{n}}}\right|>0, \\
& \inf _{n}\left|\frac{\beta_{2}}{I_{\rho}}+\alpha_{2} e^{-i \tau \sqrt{\mu_{n}}}\right|>0,
\end{aligned}
$$

we have

$$
\inf _{n}\left\|C\left(\frac{\Phi_{n}}{i \sqrt{\mu_{n}}}, \Phi_{n}\right)\right\|_{\mathbb{C}^{2}}^{2}>0 .
$$

Clearly, when $\beta_{1} \neq \rho \alpha_{1}$ and $\beta_{2} \neq I_{\rho} \alpha_{2}$, we also have

$$
\inf _{n}\left\|C\left(\frac{\Phi_{n}}{i \sqrt{\mu_{n}}}, \Phi_{n}\right)\right\|_{\mathbb{C}^{2}}^{2}>0 .
$$

Therefore, the conditions in Theorem 2 are easily verified.

The control method proposed in this paper can be used to the system of output availed system by using the Luenberger observer. Also we have noted that the method is only fitting the continuous model; for the model of data-driven system (e.g., see, [18]), it might fail. So we need to study the corresponding control strategy for the data-driven system. 


\section{Acknowledgment}

This research was supported by the National Science Natural Foundation in China (NSFC-61174080).

\section{References}

[1] G.-Q. Xu and D.-X. Feng, "The Riesz basis property of a Timoshenko beam with boundary feedback and application," IMA Journal of Applied Mathematics, vol. 67, no. 4, pp. 357-370, 2002.

[2] G.-Q. Xu and S. P. Yung, "Stabilization of Timoshenko beam by means of pointwise controls," ESAIM. Control, Optimisation and Calculus of Variations, vol. 9, pp. 579-600, 2003.

[3] Z.-J. Han and G.-Q. Xu, "Stabilization and Riesz basis property of two serially connected Timoshenko beams system," Journal of Applied Mathematics and Mechanics, vol. 89, no. 12, pp. 962980, 2009.

[4] S. A. Messaoudi, M. Pokojovy, and B. Said-Houari, "Nonlinear damped Timoshenko systems with second sound-global existence and exponential stability," Mathematical Methods in the Applied Sciences, vol. 32, no. 5, pp. 505-534, 2009.

[5] A. Guesmia, S. A. Messaoudi, and A. Soufyane, "Stabilization of a linear Timoshenko system with infinite history and applications to the Timoshenko-heat systems," Electronic Journal of Differential Equations, vol. 2012, no. 193, pp. 1-5, 2012.

[6] G. Q. Xu, S. P. Yung, and L. K. Li, "Stabilization of wave systems with input delay in the boundary control," ESAIM, vol. 12, no. 4, pp. 770-785, 2006.

[7] S. Nicaise and C. Pignotti, "Stabilization of the wave equation with boundary or internal distributed delay," Differential and Integral Equations, vol. 21, no. 9-10, pp. 935-958, 2008.

[8] S. Nicaise and J. Valein, "Stabilization of the wave equation on 1$\mathrm{d}$ networks with a delay term in the nodal feedbacks," Networks and Heterogeneous Media, vol. 2, no. 3, pp. 425-479, 2007.

[9] Y. F. Shang, G. Q. Xu, and Y. L. Chen, "Stability analysis of EulerBernoulli beam with input delay in the boundary control," Asian Journal of Control, vol. 14, no. 1, pp. 186-196, 2012.

[10] S. Nicaise and C. Pignotti, "Stability and instability results of the wave equation with a delay term in the boundary or internal feedbacks," SIAM Journal on Control and Optimization, vol. 45, no. 5, pp. 1561-1585, 2006.

[11] Y. F. Shang and G. Q. Xu, "Stabilization of an Euler-Bernoulli beam with input delay in the boundary control," Systems \& Control Letters, vol. 61, no. 11, pp. 1069-1078, 2012.

[12] Z. J. Han and G. Q. Xu, "Output-based stabilization of EulerBernoulli beam with time-delay in boundary input," IMA Journal of Mathematical Control and Information, p. 1, 2013.

[13] G. Q. Xu and H. X. Wang, "Stabilization of Timoshenko beam system with delay in the boundary control," International Journal of Control, vol. 86, pp. 1165-1178, 2013.

[14] J. U. Kim and Y. Renardy, "Boundary control of the Timoshenko beam," SIAM Journal on Control and Optimization, vol. 25, no. 6, pp. 1417-1429, 1987.

[15] B.-Z. Guo and Y.-H. Luo, "Controllability and stability of a second-order hyperbolic system with collocated sensor/actuator," Systems \& Control Letters, vol. 46, no. 1, pp. 45-65, 2002.

[16] G. Q. Xu, "Exponential family, Riesz basis sequence and its applications in control theory, in preparation".
[17] Yu. I. Lyubich and V. O. Phóng, "Asymptotic stability of linear differential equations in Banach spaces," Studia Mathematica, vol. 88, no. 1, pp. 37-42, 1988.

[18] S. Yin, S. X. Ding, A. Haghani, H. Y. Hao, and P. Zhang, "A comparison study of basic data-driven fault diagnosis and process monitoring methods on the benchmark Tennessee Eastman process," Journal of Process Control, vol. 22, pp. 15671581, 2012. 


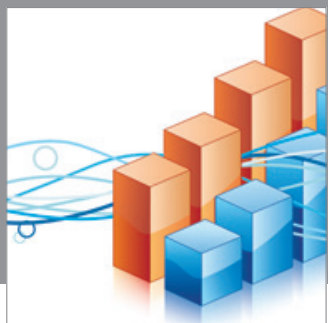

Advances in

Operations Research

mansans

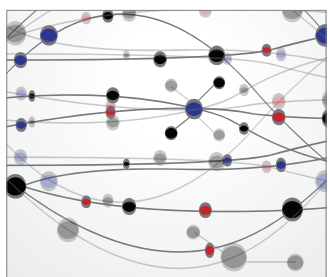

The Scientific World Journal
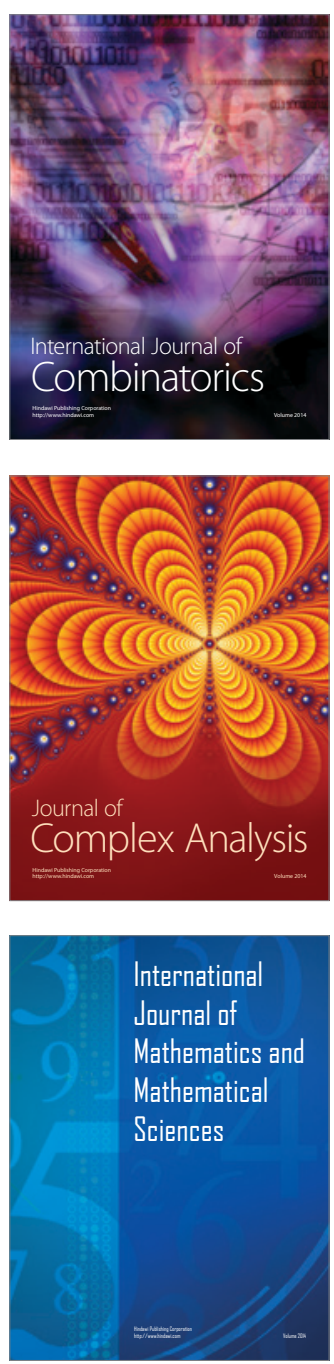
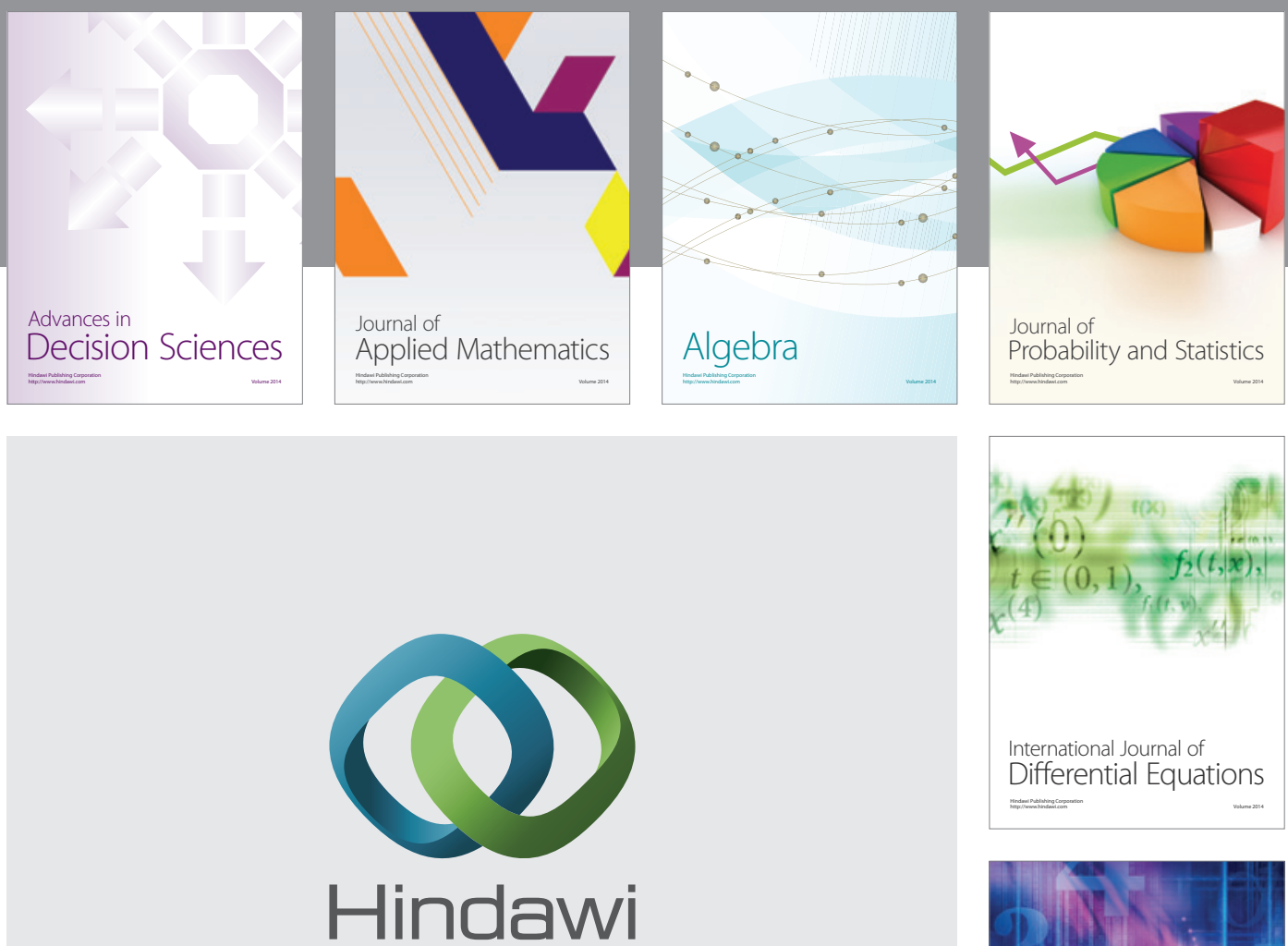

Submit your manuscripts at http://www.hindawi.com
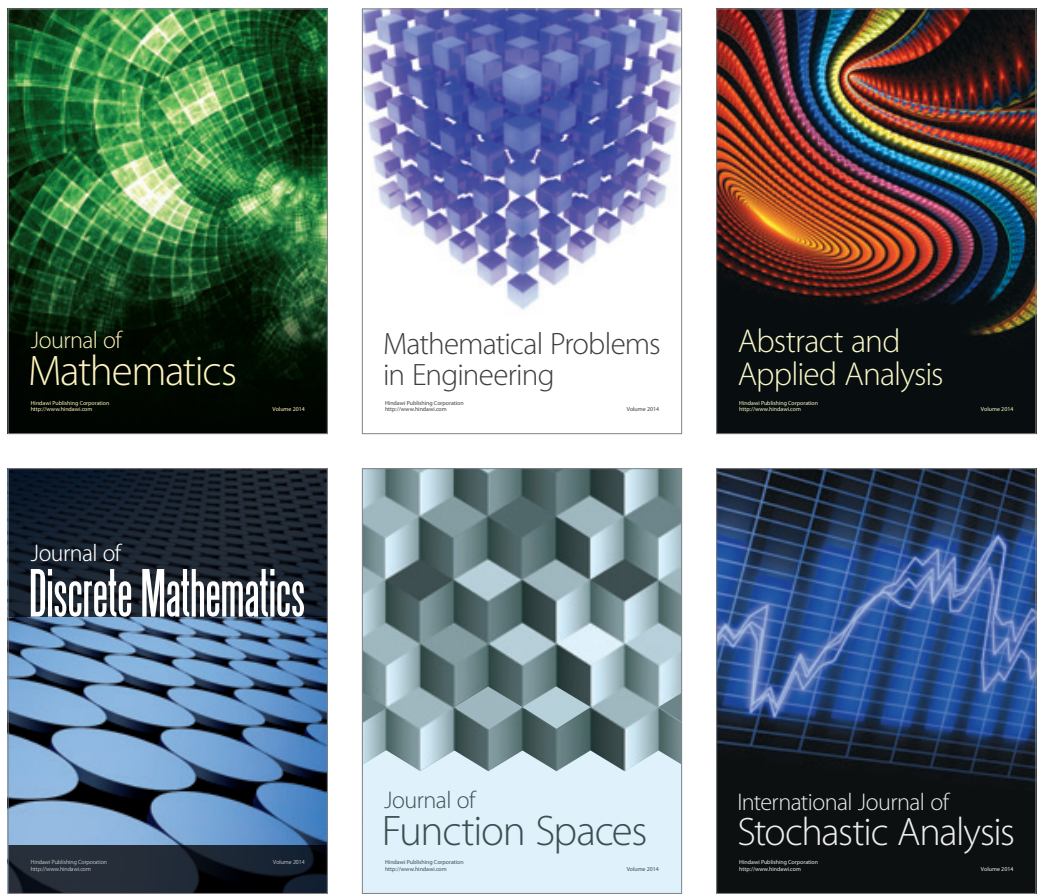

Journal of

Function Spaces

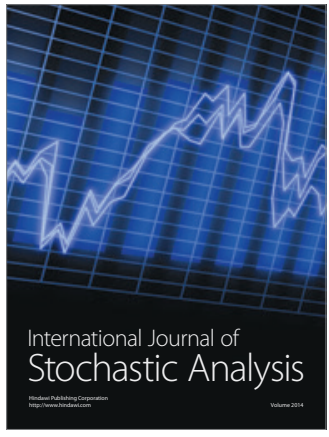

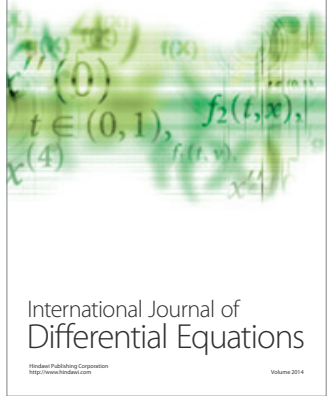
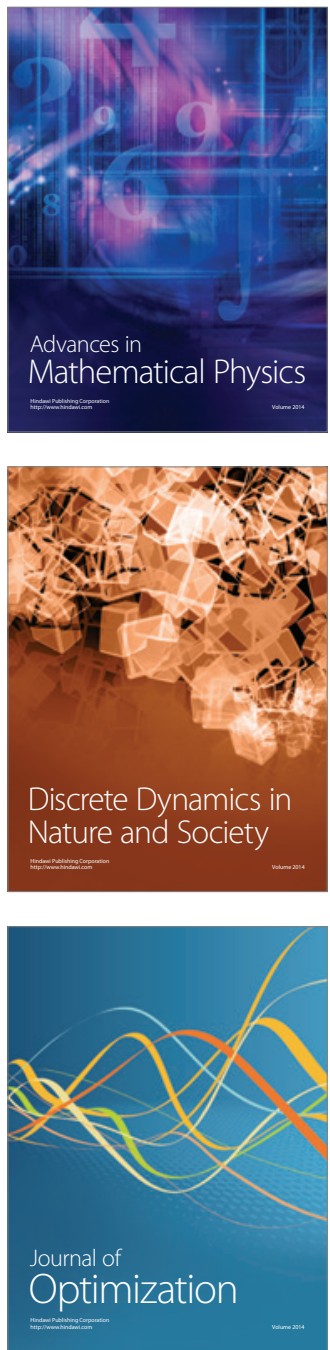